\title{
Visuomotor Transformations Underlying Arm Movements toward Visual Targets: A Neural Network Model of Cerebral Cortical Operations
}

\author{
Yves Burnod, ${ }^{1}$ Philippe Grandguillaume, ${ }^{1}$ Isabelle Otto, ${ }^{2}$ Stefano Ferraina, ${ }^{2}$ Paul B. Johnson, ${ }^{3}$ and Roberto \\ Caminiti $^{2}$ \\ "Institut des Neurosciences, Département des Neurosciences de la Vision, Université Paris VI, 75005 Paris, France, \\ ${ }^{2}$ Istituto di Fisiologia umana, Università degli Studi di Roma "La Sapienza," 00185 Roma, Italy, and "Curriculum in \\ Neurobiology, University of North Carolina, Chapel Hill, North Carolina 27599
}

We propose a biologically realistic neural network that computes coordinate transformations for the command of arm reaching movements in 3-D space. This model is consistent with anatomical and physiological data on the cortical areas involved in the command of these movements. Studies of the neuronal activity in the motor (Georgopoulos et al., 1986; Schwartz et al., 1988; Caminiti et al., 1990a) and premotor (Caminiti et al., 1990b, 1991) cortices of behaving monkeys have shown that the activity of individual arm-related neurons is broadly tuned around a preferred direction of movements in 3-D space. Recent data demonstrate that in both frontal areas (Caminiti et al., 1990a,b, 1991) these cell preferred directions rotate with the initial position of the arm. Furthermore, the rotation of the population of preferred directions precisely corresponds to the rotation of the arm in space.

The neural network model computes the motor command by combining the visual information about movement trajectory with the kinesthetic information concerning the orientation of the arm in space. The appropriate combination, learned by the network from spontaneous movement, can be approximated by a bilinear operation that can be interpreted as a projection of the visual information on a reference frame that rotates with the arm. This bilinear combination implies that neural circuits converging on a single neuron in the motor and premotor cortices can learn and generalize the appropriate command in a 2-D subspace but not in the whole 3-D space. However, the uniform distribution of cell preferred directions in these frontal areas can explain the computation of the correct solution by a population of cortical neurons. The model is consistent with the existing neurophysiological data and predicts how visual and somatic information can be combined in the different processing steps of the visuomotor transformation subserving visual reaching.

\footnotetext{
Received July 24, 1991; revised Nov. 4, 1991; accepted Nov. 19, 1991.

This work was supported by the European Economic Community (Grant SCI 0028-c/A). We are especially thankful to E. Guigon, B. Dorizzi, E. Foumier, P. Germain, and M. Dufosse for their useful ideas and suggestions concerning this study.

Correspondence should be addressed to Y. Burnod, Institut des Neurosciences Département des Neurosciences de la Vision, Université P. et M. Curie, 9 quai St. Bernard, Bât. C, 6ème étage, 75005 Paris, France.

Copyright (C) 1992 Society for Neuroscience 0270-6474/92/121435-19\$05.00/0
}

Adaptive sensorimotor behaviors are generated by neural interactions occurring in different parts of the brain and linked to different sensory and motor reference frames (Paillard, 1990). These internal interactions can be viewed as "coordinate transformations" between the corresponding frames of reference. For example, to perform reaching movements toward visual targets, the cortex must transform the visual information related to target location and the proprioceptive inputs concerning the arm position in space (both representing the "desired trajectory") into an appropriate motor command. The central representations of such visuomotor transformations, as well as the coordinate systems used by the cortex to represent planning and execution of arm movements toward visual targets, are matters of intense research. It has been proposed that, when a reaching movement is made toward a visual target, the initial motor planning occurs in the external coordinates of the physical world, encoding for example the hand trajectory in space (Morasso, 1981; Abend et al., 1982, Hogan, 1985, 1988; Hollerbach and Atkeson, 1987). A coordinate transformation from this Cartesian reference frame to muscle torques (inverse kinematics and dynamics) is then necessary to bring the hand to the desired position in space. Several intermediate steps can be described for this transformation. For example, during multiarticular motion in space, there is a constant relationship between joint angular velocities that suggests a coding of arm movement in terms of joint variables (Soechting and Lacquaniti, 1981; Lacquaniti and Soechting, 1982; Soechting and Terzuolo, 1988; see also Soechting and Flanders, 1989a,b).

At the neurophysiological level, many studies on arm movement direction toward visual targets have shown that neural activity in the motor (Georgopoulos et al., 1982, 1986; Schwartz et al., 1988; Kalaska et al., 1989; Caminiti et al., 1990a), premotor (Caminiti et al., 1990b, 1991), and parietal cortices (Kalaska et al., 1983, 1990) is broadly tuned around a particular direction of movement, the "cell's preferred direction." These results raise questions concerning the role of frontal and parietal cortices in the transformation from extrinsic into intrinsic coordinates, for the direction of movement could indeed be encoded within either an extrinsic extrapersonal coordinate system (direction of movement in space), an intrinsic corporeal frame of reference (patterns of muscle activity or joint variables), or both combined. In order to address this question, an experimental paradigm was designed (Caminiti et al., 1990a, 1991) to achieve a dissociation between movement trajectory direc- 
tions and underlying patterns of muscle activity and joint angles (i.e., between extrinsic frames of reference and intrinsic ones). In these studies, the monkey was required to perform arm movements of parallel directions within different parts of the extrapersonal space. The results showed that, in both motor and premotor cortices, the cells preferred direction changed with the initial position of the arm and that the global population of cell preferred directions had invariant properties in an arm-centered reference frame.

These neurophysiological results strongly suggest that cortical circuits compute the appropriate motor command by projecting the "visual" information about the target location on a reference frame rotating with the arm. In this respect, the frontal areas seem to possess an internal representation of space where coding of hand trajectory would occur within a coordinate system centered on the shoulder joint. Any time the arm moves to bring the hand to a desired location of the work space, the frame of reference is reset to the new coordinates of the shoulder joint. These quantitative data on neuronal activities provide an insight into the computation performed by the cortex for visuomotor coordinate transformation.

Understanding these cortical operations can now be approached by modeling, since quantitative experimental results can provide strong constraints to be used for the design of models. Several models have been proposed for the learning of arm kinematics and dynamics, each one focusing on different specific aspects of the movement. The internal operations performed by the nervous system to command arm movements can indeed be modeled, as in robotics, by an explicit computation of the torque changes from the desired trajectory. A multilayer neural network can learn this computation, using an error signal that is the motor correction command (Kawato et al., 1987, 1990). A decomposition of intermediate computational steps has been proposed in direct relation with several parts of the nervous system and based upon anatomical and physiological data (Arbib, 1981; Bullock and Crossherg, 1988; Kawato et al., 1990). More generally, it is important to mention models of coordinate transformations, from retinal to head-centered coordinate systems, since these transformations could be generalized to visually guided arm reaching movements. A simple network can learn to combine the visual input on the retina and the position of the eye in the orbit in order to produce an output that is constant in an head-centered reference frame (Zipser and Andersen, 1988). In this latter study, units in the "hidden layers" of the designed network showed receptive field structures and gating properties similar to those of neurons recorded in the area $7 \mathrm{a}$ (Andersen and Mountcastle, 1983; Andersen et al., 1987). Computation of an invariant representation of visual targets in head-centered coordinates can also be learned by networks with units directly performing bilinear combinations of proprioceptive and visual information (Kuperstein, 1988b). The appropriate sensory-motor transformations can be learned from the correlation, or "self-consistency," of sensory signals produced by spontaneous movements (Kuperstein, 1988a).

The aim of this article is, while focusing on the computation of visuomotor transformations in the initial ballistic phase of the reaching movement, to propose a simple neural network model performing a computation that fits the neuronal activities observed in motor and premotor cortices during 3-D arm reaching (Caminiti et al., 1990a,b, 1991). For this purpose, we have imposed three major constraints on this model. First, the global processing of the whole population of units must perform a transformation from visual into motor coordinates in order to produce the command orienting the movement toward the target, whatever the initial position of the arm. Second, the operations and learning properties of these basic processing units should model the functions of the basic neuronal circuit of the cerebral cortex (for discussion, see Mountcastle, 1978). Third, processing units should behave as neurons in the cortical areas involved in arm reaching movements, as premotor and motor cortices. In the following section, we will first detail these relevant neurophysiological data.

\section{Cortical Cell Activity and Arm Reaching Movements in 3-D Space: Neurophysiological Results}

The experiments performed by Caminiti et al. (1990a,b, 1991), while quantifying the relations between neural activity and direction of arm movements toward visual targets in 3-D space,

\footnotetext{
Figure 1. Neurophysiological results [reproduced with permission from Caminiti et al. (1990a,b, 1991)]. $A$, The animal sat on a primate chair 25 $\mathrm{cm}$ away from the apparatus. $B$, Within any one part (left, center, right) of the work space, the animals made arm movements in eight different directions (arrows), starting from a common central position (solid dots), toward targets located at the vertices of an imaginary cube. Across the work space, animals made eight triplets of movements of parallel directions (1-11-21, 2-12-22, 3-13-23, etc.). Numbers indicate directions of movement. $C$, Rasters of impulse activity of a premotor cortical cell studied while the monkey was performing the task. Five replications for every movement direction were aligned to the movement onset $(M)$. $T$ indicates target presentation. Longer vertical bars indicate, from left to right, beginning of the trial, target onset, movement onset, beginning and end of target holding time. Numbers on the vertical axes indicate directions of movement, as labeled in $B$. Notice the changes in firing frequency as movements of similar directions (e.g., 1-11-21, 2-12-22, etc.) were made within different parts of space. $D$, Spatial orientation of a cell's preferred direction computed from cell activity collected while the animal was working on the left, center, and right parts of the work space. Notice the rotation in space of the preferred direction vector $(P D)$. $E$, Plots of the rotation of premotor cortical cell preferred directions in the task. Each red line indicates the trajectory followed by a cell's preferred direction vector, with the tail (darkest part) indicating the position of the vector when the animal worked in the left part of space and the head (lightest part) its position when the animal performed the task in the right part of the work space. Brighter vectors correspond to preferred directions lying in the part of space nearer to the observer. $F$, Plots of the rotation of motor cortical cell preferred dircetions. Conventions arc as in $E$. Notice that in both premotor and motor cortices the dominant rotation of cell preferred directions occurs around the z-axis, that is, in the horizontal plane, which is also the plane in which the monkey's arm rotates most to perform the task. $G$, Neuronal movement population vectors computed from cell activity in the premotor cortex. Colored vectors indicate population vectors from left (yellow), center (red), and right (blue) parts of the work space. Population vectors from the three parts of the work space have been superimposed to a single cube and normalized to unit length to facilitate comparison of population vectors for "parallel" movement directions. Thus, for example, the three vectors pointing to the lower left represent the population vectors for movement directions 3 (yellow), 13 (red), and 23 (blue). The axes correspond to the $x, y$, and $z$ axes of $A$. $H$, Neuronal movement population vectors computed from cell activity in the motor cortex. Conventions are as in $G$. Notice how in both premotor and motor cortices the movement population vectors did not change significantly their spatial orientation as movements of similar direction were performed across the work space.
} 
A

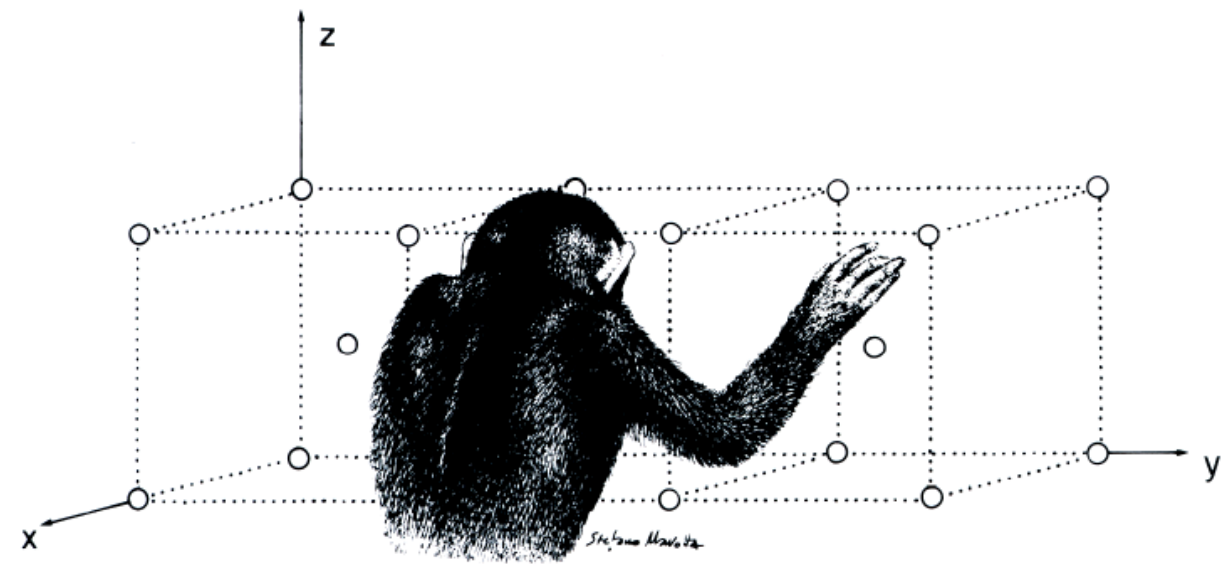

B

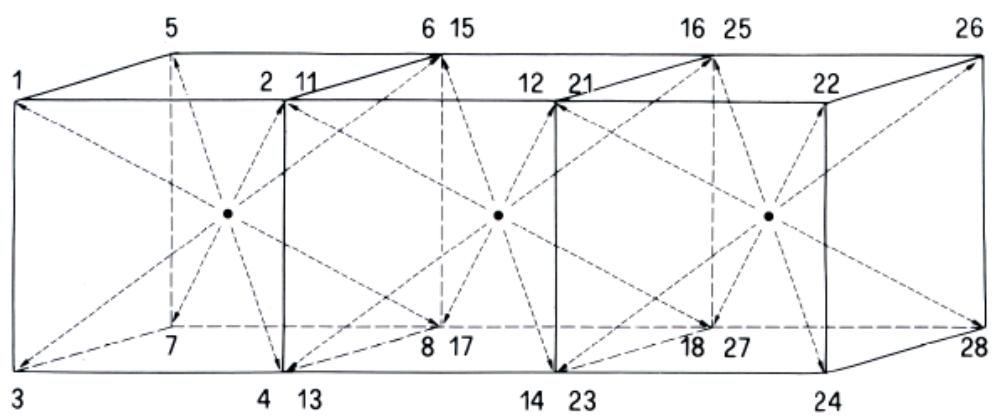

C
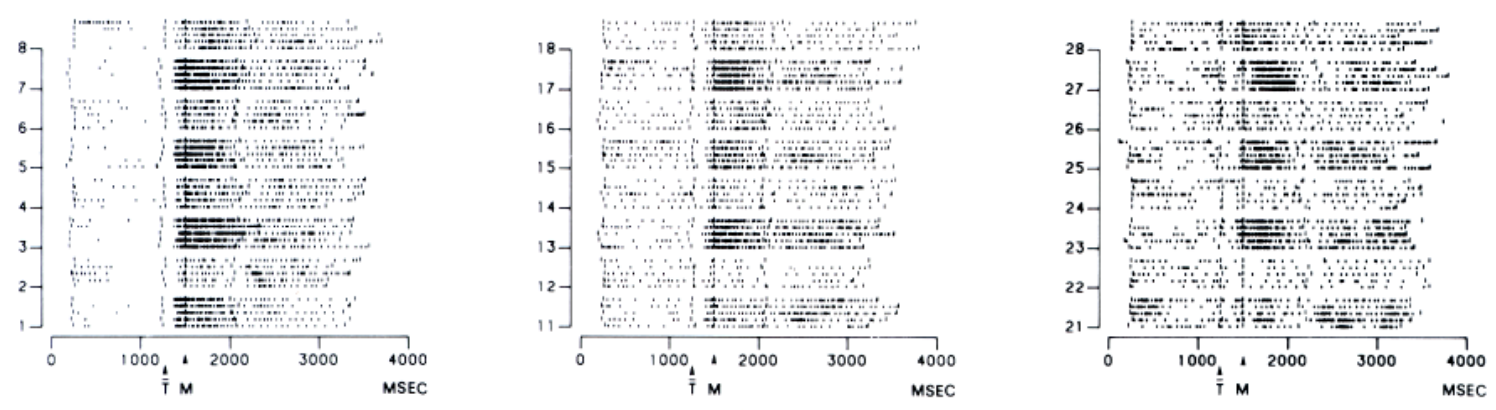

D
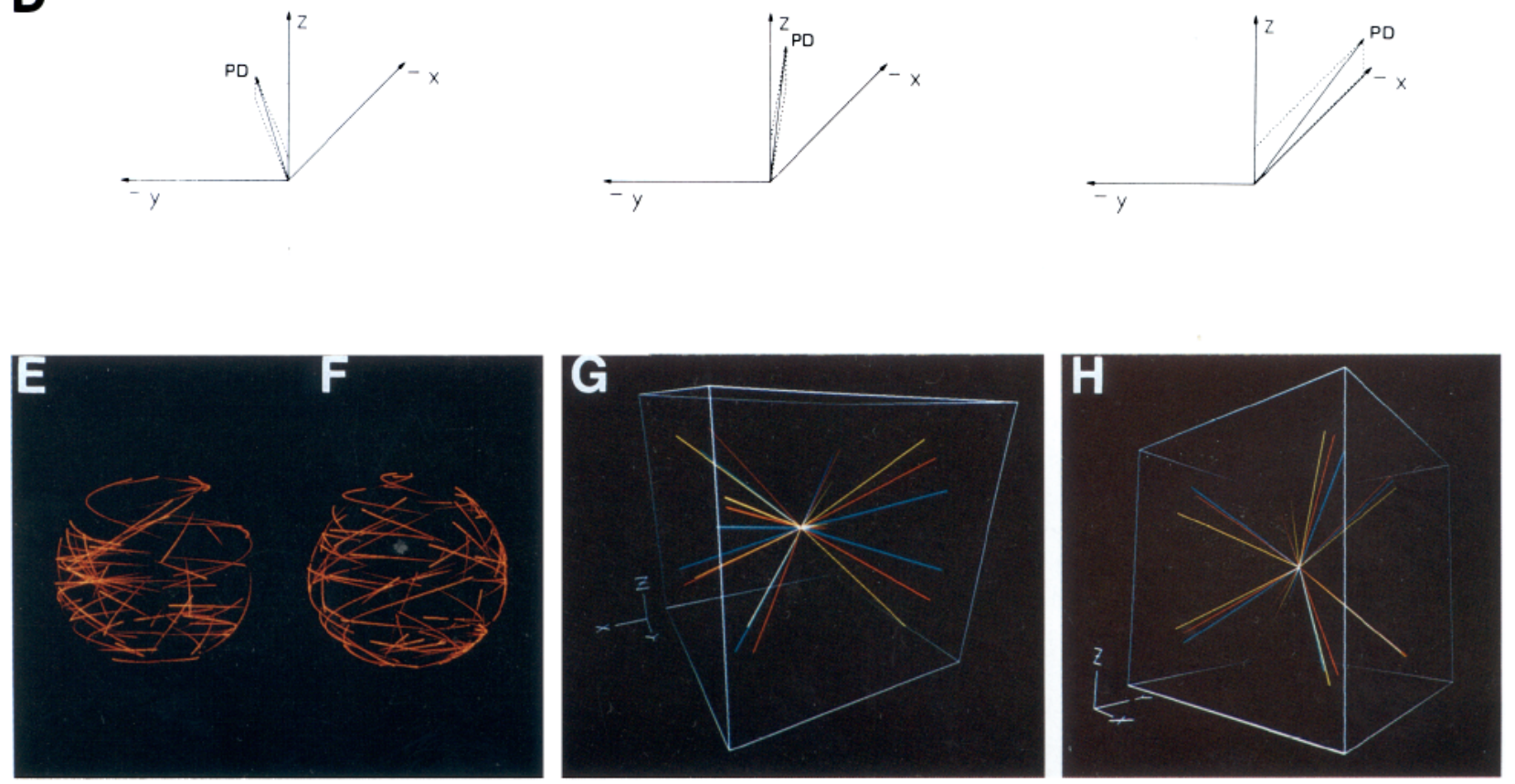
were designed to assess the coordinate system used by the cerebral cortex to code arm movement direction. In the experimental paradigm, neuronal activities were recorded in the shoulder zone of the motor (area 4) and premotor (area 6) cortices while monkeys performed arm reaching movements. These movements were made from a given initial position of the arm to a visual target determined at each trial by a conditioning program (Fig. 1A). These trajectories followed eight possible directions that could be performed in three different parts of the work space, due to three initial positions of the arm (as in three adjacent imaginary cubes). The recordings of movement trajectory showed that the hand traveled along approximately parallel paths in the three parts of the work space. A qualitative analysis for each recorded neuron determined its relation with shoulder joint movements and the presence and quality of passive inputs from skin, deep tissues, muscles, and joints. Only those cells related to movements at the shoulder joint were selected for quantitative studies. The following summarizes the results of these studies.

\section{Directional properties of cortical neurons}

In each of the three parts of the work space tested, cell activity in the premotor cortex varies in an orderly fashion with the direction of movement (Fig. 1C). This figure shows that, for a constant initial position of the arm, there is a particular movement direction for which the cell discharge frequency is maximal, namely the "cell's preferred direction" (see also Gcorgopoulos et al., 1982, 1985; Schwartz et al., 1988; Kalaska et al., 1989; Caminiti et al., 1990a). The neuronal activity for movements in other directions can be predicted by the cosine of the angle between these movement directions and the preferred one. In the premotor cortex, most arm-related cells are directionally tuned and follow this model. Similar results had been previously obtained in the motor cortex (Georgopoulos et al., 1982; Schwartz et al., 1988; Kalaska et al., 1989; Caminiti et al., 1990a) and in the parietal cortex (area 5; Kalaska et al., 1983, 1990) as well.

\section{Uniform distribution of cell preferred directions}

The spatial distribution of cell preferred directions in motor and premotor cortices was examined in each part of the work space. The set of preferred directions, expressed as vectors of unit length with origin at the origin of the movement, covered the cntirc 3-D continuum. This distribution was uniform in each of the three parts of the work space (see also Schwartz et al., 1988, for data on motor cortex).

\section{Shift of cell preferred direction with the initial position of the arm}

When the initial position of the arm was changed, the orientation of the individual cell preferred direction rotated in space, as shown in Figure $1 D$. To quantify the rotation of the whole set of preferred directions in both motor and premotor cortices, a spherical regression analysis (Jupp and Mardia, 1980) was performed between preferred direction vectors from the three parts of the work space. Although these shifts of cell preferred directions were different for different cells, at the population level, they resulted in an overall rotation (Fig. $1 E, F$ ) that exactly predicted the rotation of the shoulder joint necessary to bring the hand within the threc different parts of space.

These analyses on cell preferred directions were performed during an "experimental time," comprising both "reaction time" (from target onset to movement onset) and "movement time."
The spherical regression analysis showed that the angles of rolation of the population of preferred directions were similar during reaction and movement time, suggesting that this rotation is an expression of a central motor process exhibiting significant congruence between planning and execution. Furthermore, these results suggest that the rotation of cell preferred directions is not the result of a feedback loop reflecting the execution of the movement.

\section{Population code}

The broad relationship between cell activity and direction of movement has led to the concept that the direction of movement can be predicted by a neuronal population and, more precisely, by the computation of a "neuronal movement population vector" (Georgopoulos et al., 1983, 1986, 1988). The population vector is the vectorial sum of the contributions made by each individual neuron along its preferred direction: in the motor (Georgopoulos et al., 1983, 1986, 1988; Kalaska et al., 1989; Caminiti et al., 1990a), premotor (Caminiti et al., 1990b, 1991), and parietal (Kalaska et al., 1983, 1990) cortices and in the cerebellum (Fortier et al., 1989) as well, the direction of the effective movement of the arm can in fact be predicted by a population of neurons, summing their preferred direction vectors weighted by their own activities.

\section{Constancy in orientation of the population vector for parallel directions of movement}

When movements were made in parallel directions in the three different parts of the work space, in both premotor (Fig. $1 \mathrm{H}$ ) and motor (Fig. $1 G$ ) cortices, there was no significant rotation of the neuronal movement population vector. Thus, the population vector accurately predicts the movement direction, even if the preferred direction of individual cells changes with the initial position of the arm.

\section{Influence of arm position on cell activities}

Previous studies (Georgopoulos et al., 1984) showed that when a monkey holds its hand at various positions in 2-D space, the activity of neurons in motor cortex and in parietal area 5 varies in a linear fashion with the position of the hand in space. Such positional effects have recently been described in the motor (Kettner et al., 1988; Caminiti et al., 1991) and in the premotor cortices (Caminiti et al., 1991) by using a 3-D task. Thesc results indicate that a signal concerning the spatial position and orientation of the arm influences motor, premotor, and parietal cortical activity.

\section{Coding of direction versus final position}

Since in these experiments direction of movement and end point were confounded, an experimental paradigm was used in which the monkeys performed movements with different origins and directions but converging to a common end point. The results showed that cell activities in premotor (Caminiti et al., 1991), motor (Georgopoulos et al., 1985; Caminiti et al., 1991), and parietal cortices (Georgopoulos et al., 1985) were related to the direction of movement and not to the movement end point.

\section{Cortical Cell Activity and Arm Reaching Movements in 3-D Space: Computational Model}

\section{A. Representations of inputs and outputs}

The experimental results suggest a simple model of the computation performed by the cerebral cortex to relate the sensory 
inputs to the motor command. The model is based on several assessments on the coding of input and output information that are also compatible with other data available in the literature.

Motor command. A corticospinal neuron, at least in the motor cortex, projects to several pools of motoneurons and can thus command synergistic contractions of muscles. Furthermore, during arm reaching movements, the activity of cortical neurons is maximal along a preferred direction of movement in space (for a given initial position of the arm).

We use a simple model of the motor command that is compatible with these two sets of results: small groups of cortical neurons that are together strongly active (including shoulderand elbow-related cells) command the activation of a subset of motoneurons (projecting to different muscles) resulting in an elementary arm and forearm displacement that is a 3 -D vector applied to the hand. We call "synergy vector" this effect of an elementary cortical command on the arm and hand position. It corresponds in fact to a sum of vectors, whose orientations correspond to individual muscle axes (relative to the joint) and whose magnitudes are weighted by the amount of muscle contraction. This vector changes with the initial position of the arm in space (i.e., with the initial position of the shoulder and the initial position of the elbow). For a given position of the arm, the synergy vector of a cell is collinear with the cell's "preferred direction" that is experimentally analyzed.

Activities of neurons in the cortex before the onset of a reaching movement reflect the result of the computation performed by cortical circuits to command the trajectory of the hand in space; the activity of a neuron can thus be interpreted as the prediction made by the cortical circuits projecting to this neuron that its synergy vector points in the direction of the desired trajectory, taking into account the initial position of the arm. The more the cell is active, the more the direction of the effective trajectory of the hand in space will be close to the synergy vector.

Visual and somatic information. In order to perform the appropriate motor command, the cortical network must combine two types of information, one about the desired trajectory (or target location) and the other one concerning the initial position of the arm. The first type of information, containing the representation of the direction of the movement to be performed, is acquired through visual channels. The visual pathways can provide the position of the hand in a retinotopic space: when the cyes fixate the target, this information corresponds to a vector that expresses the initial position of the hand with respect to the target. In parallel, oculomotor and proprioceptive signals can give an information about the angle of gaze providing the position of the target with respect to the body.

The second information, signaling for the current position of the arm segments relative to the body, may come from motor and/or somatic sources. An important source is muscle spindle activity, which depends upon both central command and muscle stretch. Another source could be, at the central level, efferent copies of recently performed motor operations. Where, within the distributed motor system, the combination of these visual and somatic inputs occurs is not known. However, the data presented at the level of the primary motor and premotor cortices can be interpreted as the result of this combination.

Vectorial representation of inputs and outputs. In order to quantify the visuomotor transformation, we represent by 3-D vectors the visual, somatic, and motor information that describe the monkey's environment and behavior in the experimental setup. The visual input is represented by a "visual" vector $\mathbf{V}$, which is the vector between the two lights, one signaling for the initial position of the arm and the other one for the target. The somatic input is represented by an "arm position" vector $\mathbf{P}$, which describes the initial position of the arm. The motor output is represented by its vectorial effect, that is, the "hand trajectory" vector $\mathbf{S}_{\mathrm{ef}}$, which is the vector representing the effective trajectory of the hand in space. Since we are interested in the cortical computation that occurs before the onset of movement, we only consider these vectors during the ballistic phase of the movement, which is the result of the initial motor command, before any possible correction from sensory feedback loops.

Neuronal activity in the cortex is quantified by the cell firing frequencies across the population of cortical neurons. A cortical neuron " $\alpha$ " in the motor cortex has a firing frequency at each time $A_{\alpha}(t)$, and this output activity commands a synergistic contraction of muscles (the "synergy vector"), in a direction $\mathbf{S}^{\alpha}$. This vectorial output depends upon the initial arm position vector $\mathbf{P}$, and the cortical activity $\boldsymbol{A}_{\alpha}$ is thus considered to produce an overall effect $\mathbf{S}^{\alpha}(\mathbf{P})$ on the hand trajectory. The effective trajectory $\mathbf{S}_{\text {eff }}$ results from the summed effects of all the synergy vectors of the cortical cells weighted by their own activities.

\section{B. Rules: predictions of experimental results}

The computation which is performed by the cerebral cortex in order to command the appropriate movement of the arm toward the visual target can be expressed by two simple rules, mathematically represented in Table 1 . These two rules allow the prediction of the neuronal activities in the motor and premotor cortices before the onset of movement.

Rule I: The activity of a neuron in motor and premotor cortices, before the onset of an arm reaching movement toward a visual target, is the result of a bilinear combination of visual and somatic vectorial information.

Rule II: Considering that the activity of individual cells in motor and premotor cortices produces an elementary motor command that moves the hand in a specific direction for a given arm position, namely, the synergy vector, the whole population of synergy vectors has a uniform distribution in 3-D space.

1. The first rule predicts the activation $\left(A_{\alpha}\right)$ of a cortical neuron $(\alpha)$ during the initial ballistic phase of the movement as the result of a bilinear combination of two vectors that represent, respectively, the visual information on the desired trajectory (V) and the somatic information on the initial position of the arm (P). The coefficients of this bilinear combination (matrix $\left.\mathbf{M}_{\alpha}\right)$ are specific to each cell $(\alpha)$. This rule predicts all the experimental results on single neuronal activities:

The cell activity is maximal for a "preferred direction" of movement that depends upon the initial arm position (Table 1. Eq. 1-1 deduced from Rule 1). The bilinear operation shows that (1) when the different possible trajectories of the hand are performed from the same initial position ( $P$ constant) the cell activity is maximal for a "preferred direction" $\mathbf{D}^{\alpha}$ in $3-\mathrm{D}$ space and (2) this cell's preferred direction changes with the initial position of the arm. This preferred direction is collinear with the vectorial effect of the synergistic contraction of muscles commanded by this cortical neuron, that is, to the synergy vector $\mathbf{S}^{\alpha}(\mathbf{P})$.

At any time and for each "synergy" unit, the neuronal activity is broadly tuned around a preferred direction of movement and is equivalent to the projection of the movement trajectory on its preferred direction ( $E q .1-2$ deduced from Rule 1 and $E q .1-1$ ). For a constant initial position of the arm, the bilinear operation 


\section{Table 1. Prediction of experimental results}

Rule I: The activity $A_{\alpha}$ of a cortical cell $\alpha$ is a bilinear combination of a somatic input vector $\mathbf{P}$ and a visual input vector $\mathbf{V}$ :

Rule II: The distribution of cell preferred directions $\mathbf{S}^{\alpha}$ is uniform in 3-D space. Therefore, any vector $\mathbf{X}$ can be simply defined by:

1-1. For an initial position of the arm $\mathbf{P}$, the cell activity is maximal in a "preferred direction" $\mathbf{D}^{\alpha}$ :

1-2. Cell activity $A_{\alpha}$ is predicted by the projection of the effective trajectory of the hand $\mathbf{S}_{\mathrm{eff}}$ on the cell preferred direction $\mathbf{S}^{\alpha}(\mathbf{P})$ :

1-3. The cell preferred direction changes (from $\mathbf{S}^{\alpha}$ to $\mathbf{S}^{\prime \alpha}$ ) with the arm position (rotation $\left.\mathbf{H}_{\theta}\right)$ :

1-4. Preferred directions can rotate as the arm in 2-D subspaces [planes defined by $\mathbf{P}$ and $\left.\mathbf{S}^{\alpha}(\mathbf{P})\right]$ :

1-5. The whole population of preferred directions rotates like the arm. For two vectors $\left(\mathbf{S}^{\alpha 1}, \mathbf{S}^{\alpha 2}\right)$ symmetrical with respect to the plane of rotation of the initial position of the arm:

1-6. The population vector $\mathbf{S}_{\mathrm{pop}}$ matches the hand trajectory $\mathbf{S}_{\mathrm{eff}}$ : From Eq. 1-2 and Rule II,

$$
\begin{aligned}
A_{\alpha} & =k \mathbf{P} \cdot \mathscr{M}_{\alpha} \mathbf{V} \\
\mathbf{X} & =3 / n \Sigma\left(\mathbf{X} \cdot \mathbf{S}^{\alpha}\right) \mathbf{S}^{\alpha} \\
\mathbf{D}^{\alpha}(\mathbf{P}) & =k \mathbf{S}^{\alpha}(\mathbf{P})=k^{t} \mathbf{M}_{\alpha} \mathbf{P} \\
A_{\alpha} & =k \mathbf{S}^{\alpha}(\mathbf{P}) \cdot \mathbf{S}_{\mathrm{eff}}, \text { since } \mathbf{S}_{\mathrm{eff}}=\mathbf{V} \\
\mathbf{S}^{\prime \alpha} & ={ }^{t} \mathbf{M}_{\alpha} \mathbf{H}_{\theta} \mathbf{M}_{\alpha} \mathbf{S}^{\alpha} \\
\mathbf{S}^{\prime \alpha} & =\mathbf{H}_{\theta} \mathbf{S}^{\alpha} \\
\left(\mathbf{S}^{\prime \alpha 1}+\mathbf{S}^{\prime \alpha 2}\right) & =\mathbf{H}_{\theta}\left(\mathbf{S}^{\alpha 1}+\mathbf{S}^{\alpha 2}\right) \\
\mathbf{S}_{\mathrm{pop}} & =\Sigma A_{\alpha} \mathbf{S}^{\alpha}(\mathbf{P}) \\
\mathbf{S}_{\mathrm{pop}} & =3 / n \Sigma\left(\mathbf{S}_{\mathrm{eff}} \cdot \mathbf{S}^{\alpha}\right), \mathbf{S}^{\alpha}=k \mathbf{S}_{\mathrm{eff}}
\end{aligned}
$$

Table 1 shows that the experimental results can be predicted from two fundamental rules (Rule I and Rule II) that summarize the cortical computation necessary to command arm movements in 3-D space. $A_{\alpha}$ is the output activity of a synergy unit $\alpha$, $\mathrm{V}$ is a 3-D vector representing the "visual" information on the desired trajectory, and $\mathbf{P}$ represents the 3-D vector representing the "somatic" information on the arm position. $S_{\text {err }}$ is the effective trajectory of the arm, and $\mathbf{H}_{\boldsymbol{\theta}}$ represents the rotation of the initial position of the arm; $\mathbf{M}_{\alpha}$ is a $3 \times 3$ matrix of coefficients of the bilinear combination that is specific to the unit $\alpha$; $\mathbf{S}^{\alpha}$ is the synergy vector of the unit $\alpha$, and $\mathbf{D}^{\alpha}$ is its preferred direction of movement; $\mathbf{S}_{\mathrm{pop}}$ represents the population vector, summing all the vectors of the synergy units weighted by their own activities; $\mathbf{X}$ represents any $3-\mathrm{D}$ vector.

In order to simplify notations, we have used the following conventions:

- vectors and matrix are in bold characters.

- the transpose of matrices $\mathbf{M}_{\alpha}$ is denoted ${ }^{t} \mathbf{M}_{\alpha}$.

-inner product betwecn two vectors $\mathbf{X}$ and $\mathbf{Y}$ is denoted $\mathbf{X} \cdot \mathbf{Y}$ (transposition of $\mathbf{X}$ is implicit).

$-\mathbf{a}$ vector $X$ in 3 -D space is denoted $\mathbf{X}$ while its internal representation in an $n$-D space is denoted ${ }^{n} \mathbf{X}$.

- in a similar way, $\mathbf{M}_{\alpha}$ is a $3 \times 3$ matrix and ${ }^{n} \mathbf{M}_{\alpha}$ an $n \times n$ matrix.

-the constant " $k$ " represents a scaling factor (its value may be different in the different equations).

becomes linear. Since in the task, the effective trajectory $\mathbf{S}_{\mathrm{eff}}$ is collinear to the vector between two lights $\mathbf{V}$, the neuronal activity $A_{\alpha}$ is predicted by the projection of the effective trajectory $\mathbf{S}_{\text {eff }}$ on the "preferred direction" $\mathbf{D}^{\prime \prime}(\mathbf{P})$ specific to the cell $\alpha$. Therefore, due to the collinearity between the preferred direction $\mathbf{D}^{\alpha}(\mathbf{P})$ and the synergy vector $\mathbf{S}^{\alpha}(\mathbf{P})$, we can say that the neuronal activity can be predicted by the projection of the arm trajectory $\mathbf{V}$ on the synergy vector $\mathbf{S}^{\alpha}(\mathbf{P})$.

For an individual neuron, the preferred direction changes in an orderly way with the initial position of the arm within a subregion of the work space ( $E q .1-3$ deduced from $E q .1-1$ ). In a subregion of the work space, the relation between the synergy vector and the somatic vector is a linear dependency $\mathbf{M}_{\alpha}$, specific for each cell $\alpha$. Indeed, when the arm rotates (expressed by a rotation $\mathbf{H}_{\theta}$ between two arm positions), the preferred direction changes (from $\mathbf{S}^{\alpha}$ to $\mathbf{S}^{\alpha}$ ); this change is obtained by a product of three linear operations that are not commutative in 3 -D space and does not necessarily match the rotation in 3-D space of the initial position of the arm. This is consistent with experimental data indicating that individual cell preferred direction vectors do not necessarily parallel the rotation of the arm.

For each cell, there exist 2-D subregions where the preferred direction rotates exactly as the arm (Table 1: Eq. 1-4 from Eq. 1-3). When the arm moves in the direction of a given synergy vector $\mathbf{S}^{\alpha}$, the angle between the synergy vector and the arm position is constant, and the cell preferred direction rotates exactly as the arm. In these subspaces, the linear relation $\mathbf{M}_{o}$ between the synergy vector and the somatic vector is a rotation Since this rotation and the rotation of the arm have a common axis, they are commutative and the general formula that gives the shift of the cell preferred direction in 3-D space (Eq. 1-3) is simplified (Eq. 1-4): in these 2-D subspaces, the cell preferred direction rotates the same way as the arm. The motor command is unchanged if the initial position of the arm and the desired trajectory are rotating in the same way.

Thus, the model predicts that the preferred direction of a single neuron in the motor and premotor cortices will rotate like the arm only within a specific 2-D subspace and not in the whole 3-D space.

2. The second rule, which expresses that the distribution of the synergy vectors $\mathbf{S}^{\alpha}$ is uniform, is in accordance with the experimental results that show that the distribution of cell preferred directions is uniform in the 3-D space for each initial position of the arm. In other words, for any initial position of the arm, sets of cortical cells can produce all the possible combinations of muscular contractions, resulting in a uniform sampling of movement directions in 3-D space. This second rule allows the prediction of the experimental results at the level of the population of neurons.

The whole population of preferred directions rotates like the 


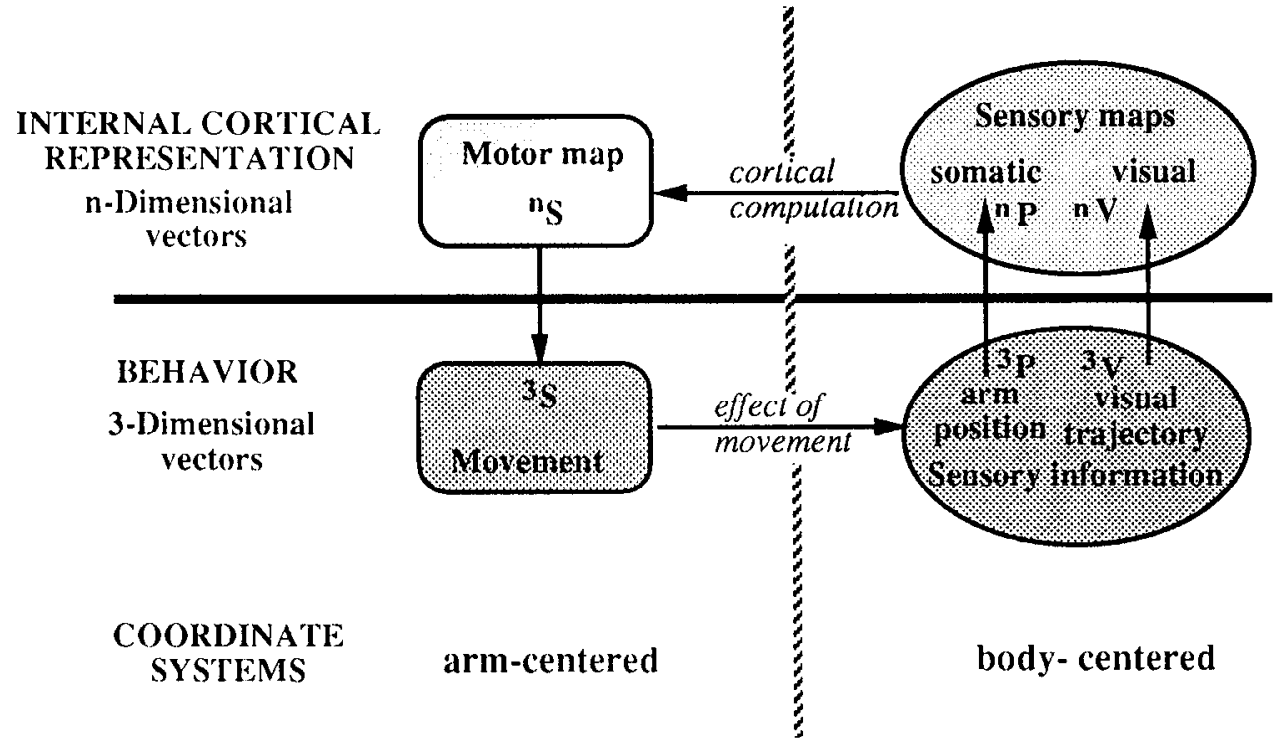

Figure 2. Relations between different coordinate systems involved in visually guided 3-D arm movements. Sensory information and motor bchavior are represented by vectors in 3-D space (the lower oval and rectangle, respectively). Each 3-D vector is "represented" in the cortex by the activity of a population of " $n$ " cells, which can be viewed as a vector in an " $n$-dimensional" space (the upper oval and rectangle). It is possible to consider these different vectors in two different reference frames: (1) a "bodycentered" coordinate system (right ovals) and an "arm-centered" reference frame (left rectangles). For further explanation, see text. initial arm position (Table 1: Eq. 1-5). The uniform distribution of cell preferred directions results in an important property of symmetry with respect to any plane. When the arm rotates in a given plane, the distribution of preferred directions $\mathbf{S}^{\alpha}$ can be partitioned in doublets $(\alpha 1, \alpha 2)$ with two preferred directions $\left[\mathbf{S}^{\alpha 1}(\mathbf{P}), \mathbf{S}^{\alpha 2}(\mathbf{P})\right]$ symmetrical with respect to this plane: due to the symmetry, the vectorial sum of these two preferred directions belongs to the plane in which the arm rotates and, consequently, rotates like the arm. Therefore, the global rotation of the whole population of preferred directions parallels the rotation of the initial arm orientation.

The population vector always predicts the movement direction (effective trajectory) even if the initial position of the arm rotates (Table 1: Eq. 1-6). The uniformly distributed synergy vectors result in an additional property: any vector is collinear to the sum of its projections on all the synergy vectors, as in 3-D space a vector is equivalent to the sum of its projections on three perpendicular axes (see Appendix A). The uniform distribution of preferred directions can thus provide an internal cortical representation of the external 3-D space with properties similar to its Cartesian representation.

The population vector $\left(\mathbf{S}_{\mathrm{vep}}\right)$ sums all the preferred direction vectors weighted by the cell activities. Since (1) the activity of each cortical neuron represents the projection of the effective trajectory on its cell's preferred direction (consequence of Rule I), and (2) the sum of all the projections of the effective trajectory on the preferred directions is collinear to this trajectory (consequence of Rule II), the population vector is always collinear to the effective trajectory. Consequently, the population vector always predicts the direction of movement, even if the individual cell preferred directions change with the position of the arm. When movements are performed in parallel directions across the work space, the population vectors should stay parallel even if they are computed from different components. The experimental results confirm this prediction.

\section{Interpretation of the rules: cortical computation}

The two rules that can predict the neuronal activities can be interpreted in functional terms: the uniform distribution of cell preferred directions (Rule II) allows a simple correspondence between vectorial information in 3-D space and its internal representation by a set of neuronal activities. The bilinear combination (Rule I) allows a dynamic transformation between two reference frames (visual and motor) that move one with respect to the other.

Internal representation of 3-D space (Rule II). Sensory and motor behavioral events occurring in the external world are represented within different cortical maps by distributed neuronal activities (Fig. 2). The 3-D vectors that describe the monkey's environment and behavior in the experimental setup are encoded within the cortical areas in a distributed fashion among $n$ cortical cells, and the whole population of " $n$ " neurons in a sensory or a motor map can thus represent an $n$-D coordinate reference frame: (1) a set of neuronal activities ${ }^{n} \mathbf{V}$ represents the visual vector ${ }^{3} \mathbf{V}$; (2) another set ${ }^{n} \mathbf{P}$ represents the arm position vector ${ }^{3} \mathbf{P}$; (3) the set of neuronal activities ${ }^{n} \mathbf{S}$ represents the cortical motor command that will result in the movement trajectory vector ${ }^{3} \mathbf{S}$.

We have seen that when the internal space is described by a specific set of uniformly distributed vectors in 3-D space (Rule II), the internal cortical representation is equivalent to a Cartesian representation of the external 3-D space (see Appendix A for the mathematical correspondence). Any transformation (e.g., a rotation) in the outside world can be represented by an equivalent transformation within the whole set of neurons. Such an internal representation is known for the motor command but is still a hypothesis for visual and somatic information. We assume that such a "3-D-like" representation is extracted in at least one of the different maps of the visual cortical system and that a similar representation exists in the somatic system.

As expressed in Rule I, the activity of each neuron is a bilinear combination of somatic and visual information. With the simple correspondence established by Rule II, this bilinear combination can also be expressed in an $n$-dimensional space (Table 2: Eq. 2-2, from Rule I and Rule II). We can thus interpret the cell activity as the output of a subnetwork that combincs (with a matrix ${ }^{n} \mathbf{M}_{\alpha}$ ) an $n$-dimensional representation of the visual trajectory $\left({ }^{n} \mathbf{V}\right)$ with an $n$-dimensional internal representation of the arm position $\left({ }^{\mathbf{P}}\right)$. This processing has similar properties in the internal representation as in a Cartesian frame of the 3-D space (see Appendix A). 


\section{Table 2. Cortical computation}

2-1. Appropriate cortical computation in an internal space:

2-2. Computation $\left(I^{s}\right)$ performed on input vector " $S$ by the lateral layer division " $s$ " of a synergy unit $\alpha$, tuned around a preferred direction of movement $\mathbf{S}^{\alpha}$ :

2-3. Computation $\left(I^{p}\right)$ performed on input vector ${ }^{n} \mathbf{P}$ by the somatic layer " $p$ " of a matching unit $(\beta \gamma)$ tuned around a given preferred arm position $\mathbf{P}^{\beta}$ :

2-4. Computation $\left(I^{v}\right)$ performed on input vector ${ }^{n} \mathbf{V}$ by the visual layer " $v$ " of a matching unit $(\beta \gamma)$ tuned, after learning, around a given preferred visual trajectory $\mathbf{V}^{\gamma}$ :

2-5. Activity of a matching unit $\beta \gamma$ after learning, due to the interlayer coefficients $(=1)$ between visual and somatic layer divisions:

2-6. Computation performed after learning by the network converging on a synergy unit $\alpha$ :

$$
\begin{aligned}
A_{\alpha} & ={ }^{n} \mathbf{P}^{n}{ }^{n} \mathbf{M}_{\alpha}{ }^{n} \mathbf{V} \\
I^{s} & =\mathbf{S}^{\alpha \bullet n} \mathbf{S} \\
I^{p} & =\mathbf{P}^{\beta} \bullet \mathbf{P} \\
I^{v} & =\mathbf{V}^{\gamma \bullet n} \mathbf{V} \\
A_{\beta_{\gamma}} & =I^{p} I^{v}=\left(\mathbf{P}^{\alpha} \bullet \mathbf{P}\right)\left(\mathbf{V}^{\gamma \bullet n} \mathbf{V}\right) \\
A_{\alpha} & =k \Sigma A_{\beta_{\gamma}} \\
A_{\alpha} & =k \Sigma\left(\mathbf{P}^{\beta} \bullet \mathbf{P}\right)\left(\mathbf{V}^{\nu \bullet n} \mathbf{V}\right)
\end{aligned}
$$

Table 2 shows how the bilinear combination computed by the cortical network to command 3-D arm reaching movements can be learned by a network of processing units; " $\mathbf{P}$ and $" \mathbf{V}$ are $n$-D vectors representing the "somatic" and "visual" information (corresponding to $\mathbf{P}$ and $\mathbf{V}$ in the 3-D space); $\mathbf{P}^{\beta}, \mathbf{V}^{\gamma}$, and $\mathbf{S}^{\alpha}$ represent, respectively, a given position of the arm, a given visual trajcctory, and a given motor command (in the $n$-D space) around which specific cells are tuned; $I^{p}, I^{v}$, and $I^{\mathrm{s}}$ represent the activation of the layer divisions (respectively, somatic, visual, and synergy) of the matching units; $A_{\beta \gamma}$ and $A_{\alpha}$ are the output activities of, respectively, a matching unit $\beta \gamma$ and a synergy unit $\alpha ;{ }^{n} \mathbf{M}_{\alpha}$ is an $n^{*} n$ matrix representing the set of coefficients of the subnetwork converging on the synergy unit $\alpha$ and tuned by learning (which corresponds to the matrix ${ }^{3} \mathbf{M}_{\alpha}$ in the external 3-D space).

Interpretation of the cortical computation in terms of coordinate transformations (Rule 1 ). A cortical command is appropriate if the effective trajectory matches the desired one in 3-D space. However, the motor information that commands the effective trajectory and the sensory information on the desired trajectory are not represented in the same reference frame: (1) The cortical command addressed to a given subset of muscles produces a constant contraction, and the vectorial effect of this contraction will turn with the rotation of the shoulder joint. Consequently, within subregions of the work space, the resulting effective trajectory has projections that are more constant in an arm-centered than in a body-centered reference frame. (2) The desired trajectory (represented either by signals on eye positions or by retinal signals on the vision of the hand when the eyes fixate the target) has more constant projections (coordinates) in a body-centered reference frame (in the experimental design, the head is fixed).

Within subregions of the work space, the operation performed by the cortex can thus be interpreted as a coordinate transformation between two different internal representations of the same 3-D vector in the external space (the trajectory of the hand). The dynamic relation between the arms and the bodycentered reference frames is given by the somatic information on the position of the arm with respect to the body. The bilinear operation (Rule I) that performs this dynamic relation suggests that the nervous system does not learn all the possible combinations of visual and somatic inputs, but it learns more general relations such as the change of the vectorial effect of the cortical command with the rotation of the shoulder joint. The circuit converging on a single cell can compute the appropriate coordinate transformation in a 2-D part of the work space (Eq. 1-4), and the whole population performs the appropriate computation in the whole 3-D space (Eq. 1-6), thanks to the uniform distribution of the cortical representation (Rule II).

\section{Cortical Cell Activity and Arm Reaching Movements in 3-D Space: Neural Network Model}

We propose a network model that is an implementation of the computational principles just described, with three main con- straints: (1) the whole network can learn the appropriate transformation between visual inputs and motor outputs for the initial phase of the arm movement, thanks to sensory-motor correspondences produced by spontaneous movements; (2) the processing units model local neuronal circuits in the cortex, with biologically plausible local rules; (3) after learning, processing units behave as cortical neurons before the onset of arm reaching movements when varying both the initial position of the arm and the direction of the movement.

\section{A. The network}

Multicellular circuits as processing units. Activation and learning properties of the processing units should not be chosen arbitrarily but should be designed to be as similar as possible to the known features of actual biological intracortical networks. The cellular tissue of the cerebral cortex is composed of a variety of neuronal assemblies sharing similar features across cortical areas. Therefore, a processing unit should model a "prototypic local circuit" of neurons rather than a prototypic neuron. We consider that such units (Burnod, 1988) do not correspond to single neurons but to cortical columns (for discussion, see Mountcastle, 1978), which are vertical neural assemblies repeated throughout different cortical areas. The term "column" refers here more to the vertical arrangement of these neurons in the depth of the cortex than to the different "surface views" obtained with different types of metabolic markers and anatomical tracers that suggest that several adjacent local vertical circuits process a variety of input-output combinations (Swindale, 1990).

Since local neuronal circuits of different cortical areas share a common architecture, we hypothesize a common global functional processing. Since these circuits consist of several cell types that have different integrative properties on specific inputs and that are distributed in separate anatomical layers, processing units have several input-output divisions modeling these cortical layers (Burnod, 1988; Alexandre et al., 1991). Each layer division corresponds to a specialized integration of subsets of inputs sharing a common origin; for example, (1) a feedforward division receives information from a sensory channel via pre- 


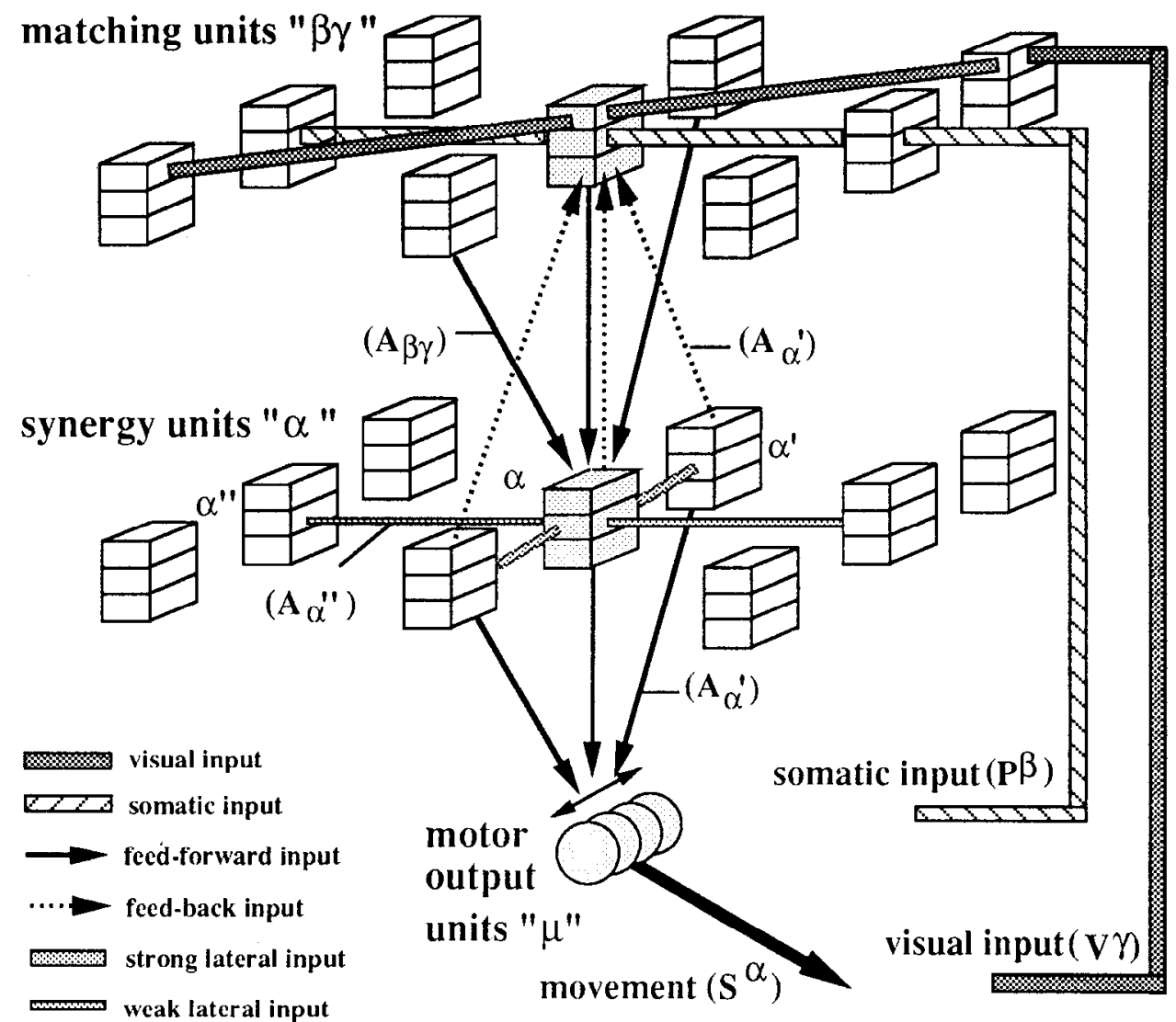

Figure 3. Architecture of the neural network modeling the operations performed by the cerebral cortex for visuomotor transformations. The processing units model the "cortical column," with inputs and outputs organized in layers. A set of "synergy" units $(\alpha)$ and a set of "matching" units $(\beta \gamma)$ are reciprocally connected. Each synergy unit addresses a motor command to a subset of "motor output units" $(\mu)$, and this command is modeled by its vectorial effect $\left(\mathbf{S}^{\alpha}\right)$ on the hand position. A matching unit receives sensory information from different sources: a "somatic input" (on its somatic layer division) that encodes the initial arm position $\left(\mathbf{P}^{\theta}\right)$, a "visual input" (on the visual layer division) that codes for the desired trajectory $\left(\mathbf{V}^{\gamma}\right)$, and feedback information (on the feedback layer division) from active synergy units $\left(A_{\alpha}\right)$. Each matching unit $\left(A_{s \gamma}\right)$ projects to synergy units. Synergy units receive a feedforward input from matching units $\left(A_{g_{r}}\right)$ a lateral input from other synergy units $\left(A_{\alpha^{\prime}}\right)$, and a feedback input from peripheral receptors (not shown in the figure). Input connections to synergy and matching units are adaptive and are tuned by the feedback loop produced by spontaneous movements. vious processing steps; (2) a lateral division represents reciprocal connections with other units of the same map; and (3) a feedback division receives an "efferent copy" of the output of a map further in the processing.

The architecture of the network. The network (Fig. 3) is composed of two successive maps of these general units: a map of "matching" units and a map of "synergy" units.

Each matching unit $(\beta \gamma)$ receives three kinds of input in three layer divisions, each one distributed among $n$ input connections (Fig. 3): the visual input $\left(\mathbf{V}^{\gamma}\right)$ represents the direction of the desired trajectory toward the visual target; the somatic input $\left(\mathbf{P}^{\beta}\right)$ represents the current initial arm position; the feedback input is composed of the outputs $\left(A_{u}\right)$ of the synergy units.

Each synergy unit $(\alpha)$ also receives three kinds of inputs in three separate layer divisions (vectors of $n$ components): a feedforward input from the matching units $\left(A_{\beta \gamma}\right)$, a lateral input from other synergy units, and a feedback input from somesthetic receptors activated by the movement (not shown in Fig. 3). Synergy units project to "motor output units" $(\mu)$ (modeling motor units in the spinal cord), and the overall effect of their activities is simply modeled by synergy vectors $\left(\mathbf{S}^{\alpha}\right)$.

Activation and learning rules of the processing units. In neural network models, operations performed by units are defined by an "activation rule" and long-term changes are defined by a "learning rule" (Rumelhart and McClelland, 1986). We use the same framework, but the layered organization of the cortical tissue requires at least two processing steps, as shown in Figure 4 (see Appendix B for the mathematical formulas):

1. First, each layer division integrates inputs from the same origin (e.g., visual), with specific "intralayer" coefficients. These intralayer coefficients can be tuned by learning, thanks to a simple Hebbian rule (Hebb, 1949), around values that correspond to the most probable input within this layer division when the global output of the unit is strongly active. These coefficients can thus store the relation between the output motor command and a reafferent input in a sensorimotor loop.

2. Then the processing unit integrates the activation of the different layer divisions with two terms: the influences of each layer through "layer" coefticients, and the nonlinear interactions between layers, through "interlayer" coefficients. These coefficients are also tuned by learning, with a process close to a generalized operant conditioning:

(i) When the output activity of the unit is always followed, through an external feedback loop, by the same reafferent input arriving to a given layer division, the input-output relation in the processing unit can represent a one-to-one sensory motor correspondence that always predicts the sensory effect of the movement, independently of any other conditions. In this case, the layer coefficient increases and the inputs in a single layer division can trigger a strong output (full anticipation of the effect).

(ii) When this reafferent activity occurs only sometimes, another condition represented by another type of input is required. If the conjunction of the two inputs has a higher probability of being related to a strong output, interlayer coefficients increase and the activity of a layer division acts as a gating signal (AND gate) or a gain signal (product) on the other input.

As a result, the network will not only learn the appropriate tuning on each input (intralayer coefficients) but also the type of combination (e.g., a gain) on the two inputs (i.e., visual and 


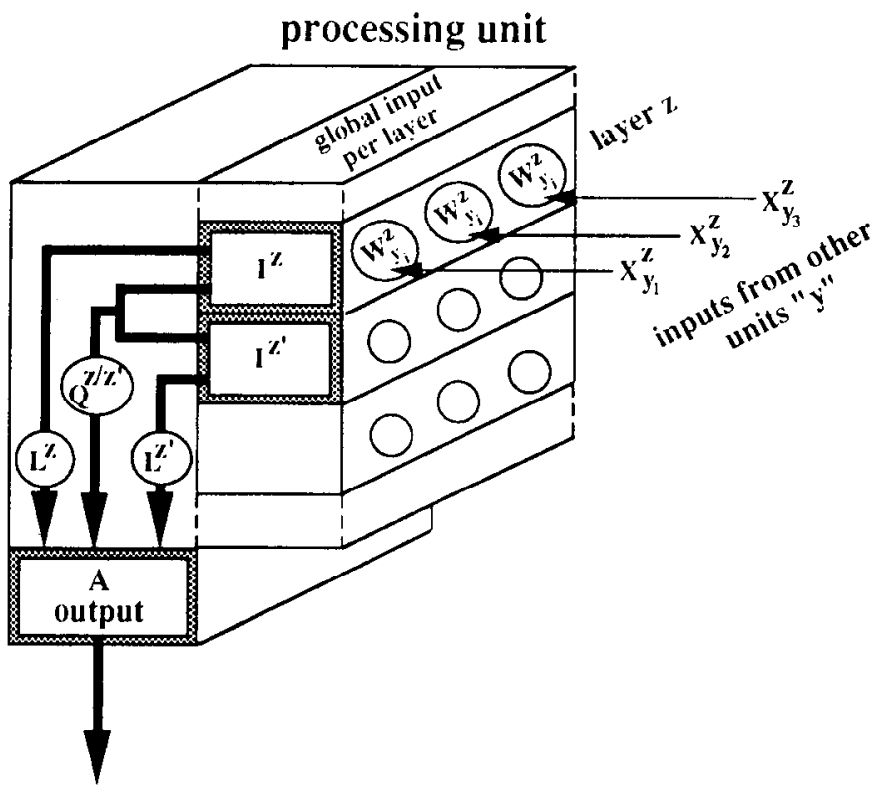

Figure 4. Processing unit modeling a cortical column. Inputs that come from the same sensory source or from other units of the same cortical map are first integrated within specific layers $\left(z, z^{\prime} \ldots\right)$ with adaptive intralayer coefficients $w_{y}{ }^{2}$. For example, in a matching unit, the layer $z$ will be the visual layer division and the layer $z^{\prime}$ will be the somatic layer division. The global inputs per layer $\left(I^{z}, I^{z}\right)$ thus obtained are then combined together with two sets of adaptive coefficients: (1) "layer" coefficients $L^{x}$, which represent the influence of each input division on the computation of the global output of the unit $A$, whatever the activity in the other layers, and (2) "interlayer" coefficients $Q^{z / z}$, which represent the mutual interactions between the different layer divisions.

somatic), necessary to produce the appropriate motor output. Learning only depends upon the probabilities of coactivation between strong inputs and outputs at the level of each processing unit. We thus distinguish strong cortical activities (high firing frequency, called the "decision" level E2) related to sensory and motor events involved in behavioral programs from lower activities (low firing frequency, called "hypothetical" level El) more related to attentional states or anticipatory processes (Burnod, 1988).

Justification of activation and learning rules. The model of the local cortical circuits outlines two basic operations: (1) cells perform a convolution-like operation on the inputs coming from the same sensory source (encoding, e.g., the orientation, the direction of movement, the spatial frequency of the stimulus) as in the orientation-selective cells in the primary visual cortex (Hubel and Wiesel, 1968)-this is modeled at the level of each layer division by a process similar to those of most of the "neural units" in the classical neural networks (weighted sum); and (2) cells can give strong nonlinear responses to the interaction of two independent sources of information (see, e.g., Andersen et al., 1987). A simple model would be a combination of AND and OR functions when considering all-or-none activities (see, e.g., Fukushima, 1980) or a combination of products and sums, as performed by sigma-pi units (Rumelhart and McClelland, 1986). Since our units model multicellular circuits, it is possible to assume that different types of cells in a column can effect different types of computation, closer either to sums or to prod- ucts, depending upon their membrane properties. ${ }^{1}$

The basic assumption for learning is that all neurons can change their synaptic weights, as in most neural networks, but with two effects: (1) within layer divisions, to adapt the tuning on each type of input, and (2) between layer division, to adapt the combination (sum, product) between inputs arriving from different sources. Learning properties of neurons are assumed from the results on associative long-term potentiation in the hippocampus (see, e.g., Barruonuevo and Brown, 1983), which show the Hebbian properties of activity-dependent changes at the cellular level (Iebb, 1949), depending upon the cooccurrence of inputs and outputs. But the rules differ from those rules used in most models since (1) there are two types of coefficients (intralayer and interlayer coefficients) corresponding to two levels of integration based on the layered architecture of the cortical tissue, (2) the temporal logic is closer to operant conditioning than to Pavlovian conditioning (strong output followed by a reafferent input), ${ }^{2}$ and (3) coefficients are adjusted to conditional probabilities that are the relative number of "excitatory" and "inhibitory" cooccurrences, based on the fact that input-output transmission in a cortical column depends upon competing excitatory and inhibitory pathways (see review in Mountcastle, 1978). If only excitatory patterns are present (always), excitatory pathways are reinforced and, after learning, a moderate input alone can trigger a strong activity in the column, even if other inputs are not active (OR gate between inputs). In the reverse case (never), only inhibitory pathways are reinforced. In the intermediate case (sometimes), both competing pathways are reinforced and, after learning, a moderate cortical input alone cannot produce a strong external output but can "gate" the efficiency of other inputs (AND gate).

\section{B. Learning}

Previous learning. To perform visuomotor transformations, the neural network has to learn the relation between the two inputs, somatic and visual, and the appropriate motor command output. These relations can be learned both during spontaneous movement and, by error correction, during visually triggered movements.

We focus on learning with spontaneous movements, as a particular stage in a general developmental scheme. We consider that successive stages correspond to different adjustments of input-output relations within and between layer divisions of processing units (Burnod, 1988). Before the learning stage based on the visuomotor loop, at least two learning steps have already independently organized motor outputs and visual inputs:

(1) Lateral connections between synergy units are already tuned by learning. The learning rules predict that the strength of re-

\footnotetext{
1 Large cells can act as coincidence detectors (low membrane conductance, short time constant, high threshold). In these cells, the probability of firing an action potential is highly dependent upon the probability of having spikes in several input channels within a short time interval; if the two inputs are independent this probability is mainly related to the product of the probabilities (one for each input channel) and consequently to the product of their firing frequencies. Conversely, small cells (interneurons, pyramidal cells of the upper layers) can merely sum the input firing frequencies (due to a higher membrane conductance, a longer time constant, and a lower threshold): the amplitude of the postsynaptic potentials reflects a time integral of input spikes and consequently reflects the firing frequency. Since the output frequency depends upon the sum of the EPSPs, the global inout operation is closer to a summation of the firing frequencies.

${ }^{2}$ These rules at the cellular level are consistent with the voltage properties of glutamate receptors (NMDA; see, e.g., Nowak et al., 1984). These receptors, and thus glutamate inputs, become more efficient when the cell is first depolarized (calcium accumulation and potentiation), that is, when a strong output is followed by a reafferent input.
} 
Synergy unit

$(\alpha)$
Matching unit

$(\beta \gamma)$

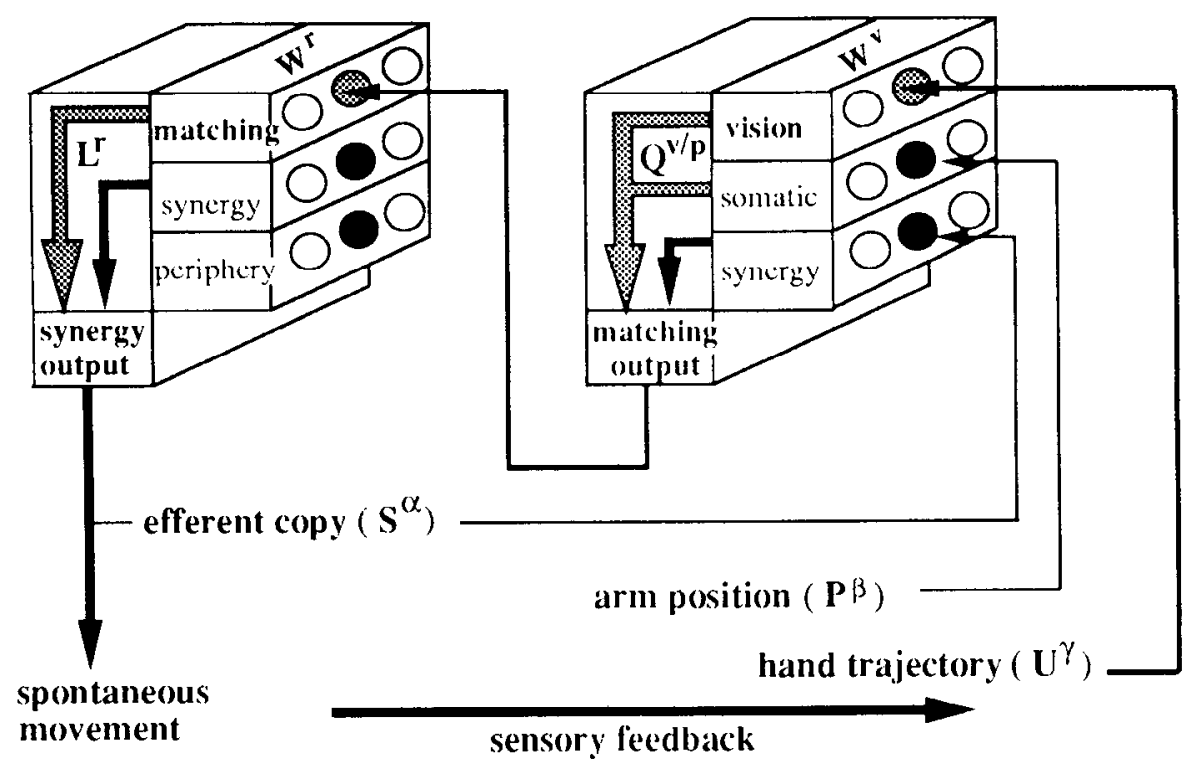

Figure 5. Tuning of adaptive coefficients through the sensory effects of spontaneous movements. This diagram shows the input-output relations of two related units, a synergy unit $(\alpha)$ and a matching unit $(\beta \gamma)$, during a spontaneous movement. Coefficients that are already tuned (prewired or tuned by a previous learning) are shown by solid circles and arrows. Coefficients that will be changed by the sensory motor loop are shown by shaded circles and arrows. In a spontaneous movement, a strongly activated synergy unit addresses both a motor command toward motor units $\mu$ and an efferent copy of this command to the matching units. Some matching units that receive this efferent copy are already gated by the information on the initial arm position. In their division, receiving visual information, the "intralaycr" coefficients will change as the inputs, which precisely represent the visual image of the effective trajectory of the hand. The operation for output computation performed by matching and synergy units will also change with learning (stippled arrows): the coefficients between the somatic and the visual information will be strong, since only the appropriate combination of somatic and visual inputs is related to a strong activity in the synergy units.

lations between two synergy units will be adjusted to the probability that the strong activation of the first one produces the same motor effect as the second one. The lateral influence between these units will thus be adjusted by learning to the cosine of the angle between the two corresponding synergy vectors (as in Eq. 2-2). This relation is relatively independent of the initial position of the arm. Connections between synergy units that share common muscular targets will be very strong, and all these cells will form a functional assembly that can trigger a specific motor effect.

(2) The visual signals are processed by several visual maps, and we consider that the visual inputs that feed the matching layer have acquired two properties through previous learning stages (see Discussion): (i) neural responses are stronger when the retinal image of the hand moves toward the fovea and when the eyes fixate a point in space; (ii) they code directional properties in 3-D space, both for the relative position of the hand with respect to the fixation point $\left(V^{\gamma}\right)$ and for the movement toward it $\left(\mathbf{U}^{\gamma}\right)$, in the same coordinate system. Visual signals that encode the relative position of the hand and the fixation point will then activate cells in anticipation of visual inputs monitoring the effective trajectory of the hand toward the fixation point.

As illustrated in Figure 5, spontaneous movements generate the repeated conjunction of three inputs at the level of a processing unit: (1) a "somatic" signal indicating the initial position of the hand $\left(\mathbf{P}^{\beta}\right)$; $(2)$ a motor command and the efferent copy of the motor command $\left(\mathbf{S}^{\alpha}\right)$; (3) a visual feedback due to the vision of the hand during movement (called $\mathbf{U}^{\gamma}$ ). The distributed learning rules predict an adaptation of tuning properties both within and between layer divisions.

Tuning properties within layers. Specific tuning properties within layer divisions of processing units can be either prewired or learned. If a bimodal map has units that are a priori independently tuned for two types of information and receive a third input, the conjunction of all the three inputs will adjust the tuning on this third one, since intralayer coefficients are shaped by the most probable input within this layer when the global output of the unit is strongly active. There are two possibilities:

(1) Either the matching layer is a bimodal map already tuned for couples $\left(\mathbf{P}^{\beta}, \mathbf{S}^{\alpha}\right)$ of "positional" and "synergy" information (Eqs. 2-2 and 2-3): the conjunction of all three inputs $\left(\mathbf{S}^{\alpha}, \mathbf{P}^{\beta}\right.$, and $\mathbf{U}^{\gamma}$ ) on the matching unit will modify, by learning, the weighting of the visual signal. The adjustable coefficients will thus converge toward the specific values corresponding to the direction of the spontaneous movement ( $\mathbf{U}^{\gamma}$; Eq. 2-4). Therefore, after learning, the unit will be tuned for the actual position of the target (or the desired trajectory) in the direction of the synergy vector.

(2) Or matching units are a priori tuned for couples $\left(\mathbf{P}^{\beta}, \mathbf{V}^{\gamma}\right)$ of "positional" and "visual" information: learning will adjust the relation with the map of synergy units. A given motor output (and thus activation of the same synergy units) corresponds to several combinations of visual and somatic information that are computed by different matching units. As a result of learning, synergy units will perform a weighted averaging on outputs of 

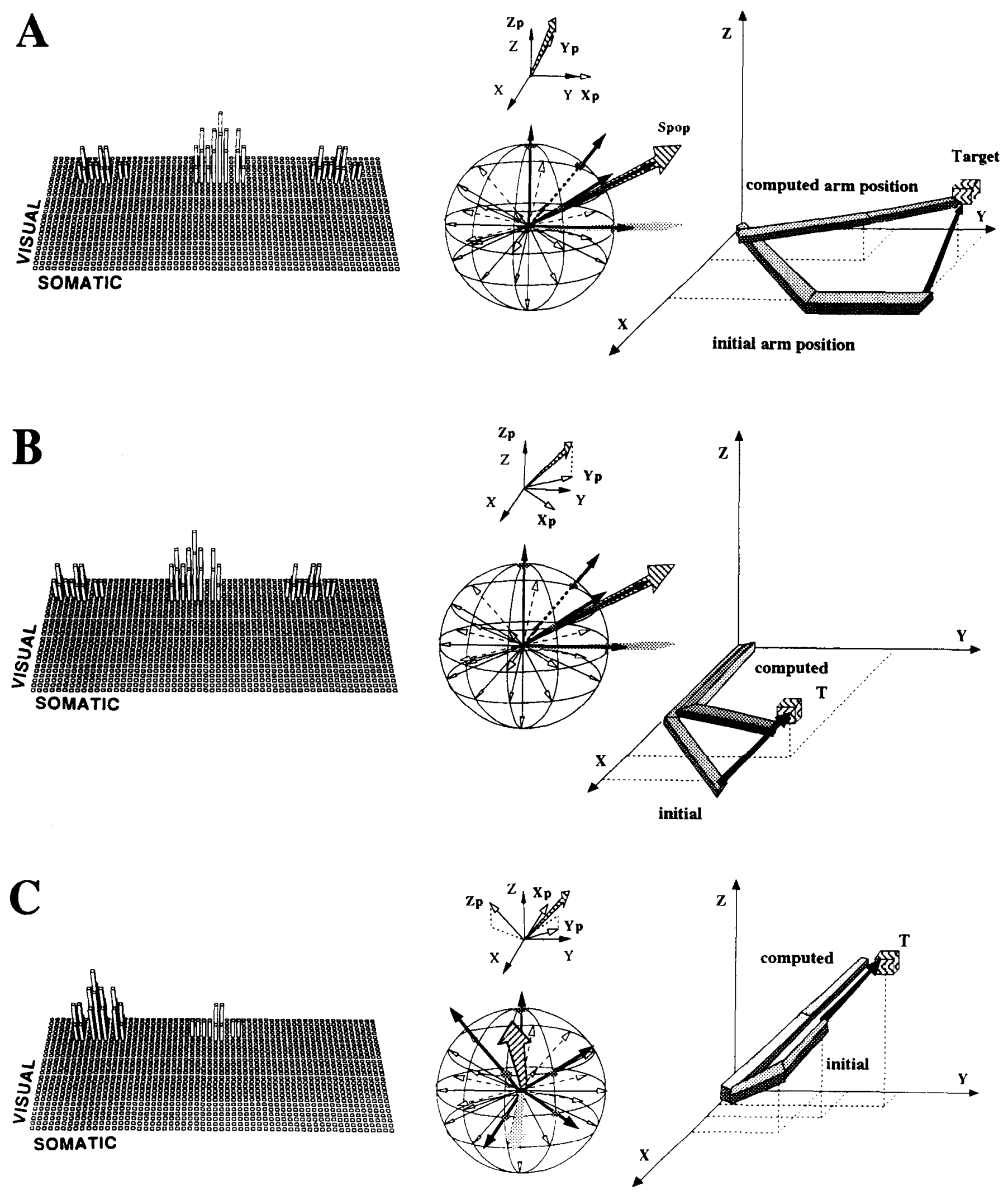

Matching units

Figure 6. Invariant properties of the cortical command for reaching movements. This figure shows the results of the network computation after learning. Three initial conditions are shown $(A-C)$. For each movement, (1) the left part shows the activation of the map of matching units; each 
the matching units extracting the appropriate combinations.

Interaction between layers. The network will also lcarn the adequate type of combination (e.g., a "sum" or a "product") on the two inputs (visual and somatic) necessary to produce the appropriate motor output. During spontaneous movements, in matching units, each type of sensory input alone has a low probability of being related to the direction of movement selectively amplified by the feedback layer division (related to the synergy units $\mathbf{S}^{*}$ ). However, this probability can be maximal when both inputs are considered. The learning rules predict that each sensory input will act as a gain on the other input (Eq. 2-5).

The bilinear operation computed by the network. When both types of tuning are effected (intra- and interlayer), the activity of each synergy unit is the result of bilinear combination (i.e., a sum of products: Eq. 2-6) between the internal representation of the arm position (somatic information ${ }^{n} \mathbf{P}$ ) and the internal representation of the target information (visual information ${ }^{n} \mathbf{V}$ ). The product terms correspond to the intermodal combinations $\left(\mathbf{P}^{\beta}, \mathbf{V}^{\gamma}\right)$ learned by matching units, and the linear term corresponds to the sum of the different possible combinations leading to the same motor output learned by the synergy units. The coefficients of the bilinear combination (matrix ${ }^{n} \mathbf{M}_{\alpha}$ ) represent pairs of input vectors $\left(\mathbf{P}^{\beta}, \mathbf{V}^{\gamma}\right)$ associated by learning with the output synergy vector $\left(\mathbf{S}^{\alpha}\right)$. It is important to stress that units, in this model, do not perform this bilinear operation a priori but learn it since it is logically related to the conditional probabilities of inputs and outputs. The overall operation learned by the network is equivalent to the computational solution (Rule I equivalent to Eq. 2-1), and consequently units in the synergy map behave like cells in motor and premotor areas.

Generalization. An important property of this type of learning is that, after a limited number of trials, the solution is generalizable to any position in spacc. For cach possible syncrgy unit, the number of matching units necessary to perform the computation depends upon the precision of the positional information. In motor, premotor, and parietal cortices, the activity of cortical cells related to the arm position shows a gradient that seems to cover large portions of space and the whole sampling could thus be accomplished by a small number of cells.

The set of learned coefficients $\left(\mathbf{M}_{\alpha}\right)$ is an internal representation of the movement produced by a specific motor command $\left(A_{\alpha}\right)$. It defines an operation that transforms the information about the target $\left(\mathbf{V}\right.$; through the operation $\left.\mathbf{M}_{\alpha} \cdot \mathbf{V}\right)$ and matches the result with the information concerning the initial arm position (by projecting $\mathbf{M}_{\alpha} \cdot \mathbf{V}$ on $\mathbf{P}$ ). This operation can be interpreted as a prediction of how a specific movement transforms an initial position into a final one (or into an appropriate trajectory). The operation that is learned represents a rotation regardless of the information source. For example, when using the angle of gaze information to define the target as a position in space, the transformation that is learned expresses the angle between two vectors representing the initial and the final position of the arm. Alternatively, by using retinal information about the position of the hand when the eyes fixate the target to define a desired trajectory, the learned operation is a transformation that expresses the angle of a trajectory vector with respect to the arm segments.

It is important to note that the shaping of the motor command at the level of the synergy units is not uniquely determined by the inputs from matching units (feedforward connections) but also by their lateral connections: even if a small number of synergy units are activated by feedforward connections, the whole population will be activated by the lateral connections in a manner proportional to the cosine of the angle between their synergy vectors and the synergy vectors of the units already active (or by the strongest ones if there are competitive inputs).

\section{Simulation}

A simulation has been performed in order to illustrate the computational principles exposed in section II and the properties of the network exposed thus far in section III. The results are shown in Figure 6 (for three initial conditions, Fig. 6A-C). A simplified arm made of two articulations, shoulder and elbow, moves in 3-D space (right part of Fig. 6) and is controlled by a network that has to learn the initial command of the muscular synergies ncccssary to move the hand toward a target. As already described, this network is made of matching units (maps in left part of Fig. 6) and synergy units (illustrated by the synergy vectors in the central part of Fig. 6).

Synergy units: sampling of the 3-D space. Each synergy unit commands an elementary arm displacement modeled in a simplified way, by a 3-D synergy vector that is applied to the hand (a cortical command is thus viewed as an elementary coordination between shoulder and elbow), and has constant coordinates in an arm-centered reference frame.

small square is a unit and the vertical bars are proportional to their activation; the map of matching units has two axes for the two independent sensory inputs, visual vectors represented within a subset of 26 reference trajectory directions (given by the rows of the map), and somatic vectors (shoulder and elbow angles) represented within a subset of 60 reference arm positions (given by the columns in the map); (2) the central part shows the spheres of synergy vectors and the activation of synergy units (solid vectors) in an internal space, with each synergy unit in the same place (and thus in an arm-centered reference frame); the axes just above the spheres show how the arm-centered reference frame (Xp,Yp,Zp) is rotated with respect to the body-centered reference frame $(X, Y, Z)$; the resulting population vector $S_{\text {vum }}$ is illustrated by an outlined hatched vector, both in the spheres of synergy units and with respect to the body-centered reference frame (above the spheres). The projection of this population vector on the plane $X p . Y p$ is also represented; (3) the right part shows, in a 3-D body-centered coordinate system $(X, Y, Z)$, the initial position of the arm, the desired trajectory (solid vector) toward the target, and the resulting position of the arm, as produced by the command computed by the network.

$A$ and $B$ represent conditions where, for two different initial positions of the arm and two different visual trajectories, the set of synergy units involved in the motor command is the same in the internal cortical representation. The population vectors are the same in the internal space and rotate like the arm in a 3-D external space (axes above the spheres), exactly as the two trajectories of the arm. In order to follow the transformation, consider that the population activities are in both cases in the plane $Y p . Z p$ (and thus perpendicular with $X p$ ); $X p$ is collinear to the forearm and $Y p$ perpendicular; consequently, the computed trajectory in the external 3-D space will turn (between $A$ and $B$ ) as $Y p$, perpendicular with the forearm.

Conversely, when comparing $B$ and $C$, which correspond to the same trajectory vector, from two different initial positions of the arm, notice the expected difference in the motor command at the level of the synergy units in the internal space (spheres). Synergy units that were maximally activated for the first arm position show less activity in the second one (as the position of the arm has changed, the synergy vector in the 3-D external space has rotated and no longer corresponds to the desired trajectory). However, even if the pool of synergy units involved in the motor command changes from conditions $B$ to $C$, the population vector does not change in the 3-D external space (axes above the spheres), since the two vectors representing the desired trajectories are parallel in the two movements (as shown in the right part). 
The spheres in the central part of Figure 6 represent the population of synergy vectors. The simulation is performed with a set of 26 vectors, uniformly distributed in 3-D space with an angle of $\pi / 4$ between two neighboring vectors. At any time, the elementary arm displacement produced by a synergy unit in the direction of its synergy vector is proportional to its activation (shown by solid vectors in the spheres of synergy vectors, Fig. 6 , center part). There is a trade-off between the number of synergy vectors and the precision of the activities of the synergy units: in the simulation, these activities are discretized in five levels. The motor command is due to a population code and results from the summed activity of all the synergy units in the direction of the synergy vectors, as expressed by the population vector $\left(\mathbf{S}_{\mathrm{pop}}\right.$, shown as an outlined hatched vector, with its projection on the horizontal plane).

In order to compare the network activity in different initial conditions (Fig. 6A-C): (1) the spheres of synergy vectors are aligned with the synergy units in the same place; these spheres can thus be viewed as a representation of the map of synergy units, with its population vector in an arm-centered reference frame, and consequently a representation of the motor command in an arm-centered reference frame; (2) the axes above each sphere show the position of the arm-centered reference frame $(X p, Y p, Z p)$ with respect to the body-centered reference frame $(X, Y, Z)$; it is thus possible to see the position of the population vector (hatched vector) in the body-centered reference frame (resulting in the effective trajectory) and to comparc it with the desired trajectory (solid vector shown in the right part): the command computed by the network is appropriate if these two vectors are parallel.

Matching units: tuning for visual directions and arm positions. As previously described, the synergy units receive information from a map of matching units that are tuned for visual and somatic information (the corresponding intralayer coefficient is preset), and receives a feedback from the synergy units. In Figure 6 (left part), the matching units are ordered with respect to two axes representing their preferred tuning for the two independent sensory inputs: (1) when the eyes fixate the target, the visual information on the initial direction of the desired trajectory is coded in a simplified way by its projection on a set of 26 reference directions (forming a uniform sampling of 3-D space); (2) the information about the initial position of the hand with respect to the body is coded by the projections of arm segments on a set of 60 reference positions (columns of the map), which combine a shoulder angle (centered on the shoulder) and an elbow angle (centered on the elbow). There are thus $26 \times 60$ matching units. In a real cortical map, the two types of inputs (somatic and visual) could be ordered in a different way, but the properties of the model are not dependent upon these relative spatial locations. Somatic inputs on the arm posilion activate a subset of matching units (subset of rows of the matching map, at left), and visual inputs on the image of the hand (when the eyes fixate the target) activate another subset (subset of columns of the matching map). The output activity of each matching unit (shown as a vertical bar in Fig. 6) results from these two inputs through two layer coefficients and one interlayer coefficient. These coefficients can change with learning and have initial values close to 1 for interlayer coefficients and close to 0 for layer coefficients. As for synergy units, matching units have a graded activity with five possible levels (as illustrated by vertical bars in Fig. 6). Output activities of these matching units activate each synergy unit through $(26 \times 60)$ intralayer coeffi- cients: the resulting activity in synergy units is shown by solid vectors in the spheres of Figure 6.

Learning sessions. The learning-dependent changes of coefficients between the matching units and the synergy units do not depend upon their initial value. A spontaneous activity is generated in the synergy map and produces a vectorial effect on the hand position that is a combination of elbow and shoulder rotations. This spontaneous movement produces a strong activation of some matching units due to the coactivation of a visual reafferent input (3-D direction of the hand) and a somatic input (position of the arm in a region of the work space, relative to the body).

As specified in the learning rules (see Appendix B), only trials when there are both a maximal activation of a synergy unit and a maximal reafferent input (movement toward the fovea) result in learning (maximal level called "E2" in learning rules). Only these optimal conditions $(60 \times 26)$ are taken into account. A single trial in each condition is sufficient, since repetition of similar conditions does not change the learned coefficients (due to conditional probabilities).

First, since the intralayer coefficients in matching units are pretuned for visual and somatic inputs, the coefficients between synergy and matching units (intralayer) store the optimal coactivations between these two inputs and the spontaneous (and thus independent) motor output. As expected from the learning rules, the layer coefficients in the matching units for either visual or somatic inputs do not increase and remain close to zero, since the relative number of occurrences of each specific sensorimotor relation is inversely related (1) to the number of possible initial positions for the visual inputs $(1 / 60)$, and (2) to the number of possible trajectories for the somatic input $(1 / 26)$. Conversely, the interlayer coefficient is stabilized to one.

Computation after learning. After learning has occurred for $60 \times 26$ reference positions (linked with maximal activations), we verify that for every initial condition (e.g., conditions A, B, and $C$ in Fig. 6), the network computes the appropriate trajectory of the hand toward the target (i.e., the population vector is parallel to the desired trajectory). The accuracy of this command is not only related to the precision of the sampling of the different internal spaces (synergy units, trajectory and position sampling), but also to the learned bilinear approximation of the optimal solution.

The three initial conditions shown in Figure 6 illustrate the properties of the learned visuomotor transformation and the predictions of the model: (1) when the desired trajectory rotates with the initial position of the arm (comparison between movements A and B), the activation of the synergy units is similar and generates a constant command in an arm-centered reference frame, even if the two sensory inputs have changed; (2) when the desired trajectories are parallel (comparison between movements $B$ and $C$ ), the individual component synergy vectors change (with the initial position of the arm), but the resulting population vectors are the same in a body-centered reference frame. Figure 6, $A$ and $B$, shows that two different desired trajectories with two different initial positions of the arm can result in a similar activation of a set of synergy units when the relative angles between the desired trajectory vectors and the arm position vectors are similar. Since the two internal cortical representations (sphere of synergy vectors in central parts of Fig. 6A,B) are aligned, with each synergy unit vector in the same place, it is possible to see that the network computes a similar activation of the synergy units in the two conditions, with a similar pop- 
ulation vector in an arm-centered coordinate system. However in the 3-D space, the two spheres of synergy vectors rotate as the initial position of the arm (as shown by the rotation of their axes above), and the population vector rotates as do the two desired trajectories. Figure $6, B$ and $C$, shows that two movements with parallel directions in space but with two different initial positions of the arm [like the trajectories performed within the three parts of the work space described in Caminiti et al. (1990a,b, 1991)] result in a change in the activation pattern of synergy units (comparison between the two spheres). Although the activation of each synergy unit is changed, the population vector computed by the network is the same in the body-centered reference frame (as shown in the axes above the spheres in Fig. $6 B, C$ ). The result of this simulation was expected by the model when considering two movements with two parallel trajectories and is consistent with the experimental results.

\section{Discussion}

After discussing the limitations of the model, we will treat three main issues addressed by this model: (1) the relation between reference frames, brain structures, and learning strategies for visuomotor transformations underlying arm movements toward visual targets; (2) the relation between neural activities, population code, and specific computation performed by the cortical neuronal circuits; (3) the anatomical substrata (in different cortical areas) of the operations performed for visuomotor transformations.

\section{Limitations of the model}

The model that we propose does not take into account either all the aspects of the motor command or all aspects of motor learning; it is limited to the initial steps of the visuomotor transformation underlying reaching toward visual targets. We have focused on the computation of the initial configuration of muscle activities that can initiate the movement in the appropriate direction toward the target.

The dynamics of the movement are not treated in the present model. The recent results of Kalaska et al. (1989) on the effects of changing load directions on cortical cell activities show how such a parameter can be used as a supplementary combination made by cortical circuits, with inputs (or expectations) signaling for these loads before the onset of the movement. Our model only attempts to solve a kinematic transformation, and, thus, further mechanisms should be added to compensate for limb dynamics. It is indeed possible that for the solution of the dynamics problem, differences may emerge between the operations performed by premotor and motor cortical circuits.

We have not considered how the coordination between elbow and shoulder could be learned: since the cortex learns the effect of a given motor command, this command can be either monoarticular or biarticular. Strong lateral connections between syncrgy units can form functional assemblics that can trigger coordinated elbow and shoulder movements: the interactions between neurons in precentral cortical zones controlling shoulder and elbow joint (Kwan et al., 1987) and the lateral connections (Huntley and Jones, 1991) favor the construction of such functional assemblies. The experimental data on arm reaching (Caminiti et al., 1990a,b, 1991) has suggested how the cortex can predict the effect of its command when the shoulder joint rotates. New experiments will be necessary to understand how this computation can take into account changes in elbow angle.

The model suggests that it is only within a limited region of the work space that a circuit converging on a single cell in the motor and premotor cortices can predict the correct motor command by a coordinate transformation. The well-established complications to the coordinate transformations that arise from the complex mechanics and dynamics of the arm can be taken into account by a specialization of cortical circuits for different subregions of the work space. The experimental data support this conclusion, since in the region of the work space where the animals performed the task (used in the neurophysiological experiments of Caminiti et al., 1990a,b, 1991), individual neurons in both premotor and motor cortices are not always directional throughout the entire work space: they can be "directional" in one part (and thus "vote" for the population solution in that region) and be "neutral" in another part. It is worthwhile to note that the population of preferred directions remains uniform in each spatial region, even if some cells are directional in only certain parts of the work space.

Maximal simplifications have been made to avoid considering the learning of all aspects of movements and to focus only on the step that seems critical for the initial visuomotor transformation. However, the network is adaptive in a general sense (like the cerebral cortex) and is not designed to learn only the task considered in this article. The same type of network can, in a very general way, adaptively capture the properties of the sensorimotor loops and of the "external" world: selective relations between the commands and their results, by the tuning of intralayer weights; specific operations to combine appropriately two different sources of information involved in a motor command, by interlayer coefficients. A crucial property of such a network is represented by its capacity of generalization to the maximum number of situations with minimal learning costs (time and number of weights).

\section{Reference frames, brain structures, and learning}

There is strong reason to believe that the brain contains several reference frames (body reference frame, object frame, world frame, and retinal frame; for discussion, see Paillard, 1990) representing perceptual and motor information. The overall transformations between these frames can be divided into successive steps, with regulatory feedback loops, and these steps can be related to specialized brain structures (Arbib, 1981). In this article, the model has been restricted to the cerebral cortical areas where quantitative neurophysiological data have suggested a simple mechanism of coordinate transformations from bodyto arm-centered reference frames. Other aspects of the movement, such as kinematic and dynamic parameters, can also be computed, and several possible models have been proposed for these computations (Bizzi et al., 1984; Bullock and Grossberg, 1988; Kawato et al., 1990).

Furthermore, structures other than the cerebral cortex, such as the cerebellum, obviously contribute to learning of the appropriate motor command (Arbib, 1981; Kawato et al., 1990), and the model proposed here can be extended to include these operations. In relating these different aspects, it is possible to take into account not only the anatomical and functional properties of brain structures but also a progressive learning strategy (Burnod and Dufossé, 1990). In this respect, visual feedback during spontaneous movements has been shown to be essential for learning sensorimotor coordinations (Held and Hein, 1963; Hein, 1971). A simple way to learn a sensorimotor transformation can thus be the movement dependent matching of sensory inputs, which can result in a first phase from spontaneous 
movements during critical periods of infancy ("self-consistency"; Kuperstein, 1988a). Such movements allow the association of a sensory-defined goal of the movement with the motor command necessary to achieve it, taking into account the initial conditions. Learning from spontaneous movements can be further refined by error-correcting mechanisms (Arbib, 1981) and, more precisely, by using the correcting motor command as the error signal (Kawato et al., 1990). This type of learning has been suggested to-occur in the cerebellar cortex. It is possible indeed that two types of learning (from spontaneous movements and by error-correcting mechanisms) are performed in two different neural structures (cerebral cortex and cerebellum), as a result of their inherent architectural and functional properties (Burnod and Dufossé, 1990). Studies on infants suggest that these two learning schemes could correspond to two successive phases of development (Hay, 1984). In early postnatal life (2-3 months), a baby learns to reach objects but is not able to make corrective movements for shaping the final position of the hand. When failing to touch an object of interest, he performs new attempts (from the initial position). During this period of life, the baby makes numerous spontaneous movements through which his hand is "captured" in his visual field. Later on, a 4-5-monthold child learns to correct, under visual control, the final position of its hand in order to succeed in reaching the object.

\section{Cell activities and population code}

The model proposed in this study is based on the directional properties of motor and premotor cortical neurons studied during 3-D movements toward visual targets. These cortical neurons are broadly tuned around a preferred direction of movement, and this coarse tuning suggests the existence of a population code for movement direction. We hypothesize that similar tuning properties and mechanisms exist for the somatic information concerning the position of the arm in space. For visual information, a similar vectorial representation has been used to account for the functional properties of light-sensitive neurons in area 7 (Steinmetz et al., 1987).

The model we propose is also based on a crucial property of the population that is the uniform distribution of cell preferred directions (Schwartz et al., 1988; Caminiti et al., 1990a, 1991). As shown in Appendix A, the uniformity of the distribution of cell preferred directions gives a powerful computational propcrty to the cortical system in the sense that the representation at the level of the whole population of cortical neurons is equivalent to a Cartesian representation of the extrapersonal 3-D space. Thus, the vectorial representation of movement trajectories is simply obtained from its projections onto the set of preferred directions, as a vector is obtained, in a 3-D Cartesian coordinate system, from its projections on the orthogonal axes. This Cartesian-like representation could simplify the relations of premotor and motor cortices with other brain regions more removed from the peripheral motor apparatus and that supposedly possess a similar internal construct of space. This construct of space, at the population level, represents movement trajectories, even if individual cells project to spinal motoneurons commanding muscle activity. This correspondence between internal and external constructs of space may be a way of reconciling apparently conflicting views on the functions of the motor cortex based either on a representation in terms of command of muscle activities (movement dynamics) or on a representation in terms of movement trajectories in 3-D space (movement kinematics).
The result of the computation can be explicit at the level of the population and not necessarily at the level of a single neuron (see, e.g., Sejnowski, 1986). In this model, what is explicitly computed at the level of a single "synergy" unit is a good prediction of the vectorial effect of the motor command on the hand position (the cell preferred direction) in a 2-D subspace. The correct prediction in the $3-\mathrm{D}$ space is explicit only at the level of the population of synergy units.

\section{Possible anatomical and functional substrates of the operations performed in visuomotor transformations}

The model that we propose leads to different predictions concerning the properties of synergy and matching units with respect to (1) their tuning to somatic and visuospatial information and their relations to the motor command as well as (2) the rotation of the preferred direction vectors when the initial position of the arm changes.

The quantitative behavior predicted for the synergy units is precisely observed in both motor and premotor cortical neurons. First, cells are tuned for a preferred direction of movement and, at the single cell level, the preferred direction rotates when the initial position of the arm changes. Second, the whole population of preferred directions predicts the rotation of the arm in space. Motor cortical cells when studied in different behavioral conditions can also integrate other types of inputs that we have not considered in this model, as shown, for example, by the interaction between force and direction in the infragranular layers of area 4 (Kalaska et al., 1989).

Properties of the matching units are a prediction of the model. Where, within the cortex, neurons with properties similar to those of matching units are localized is not known, and we can only speculate on the basis of the known functional properties of neurons recorded in those cortical areas involved in visually guided arm movements: parietal, premotor, and motor areas. Matching units, in this model, are tuned to somatic information on the initial arm position, to visual information indicating a direction of movement in 3-D space, and to active movements (through an efferent copy of the motor command). The information about the position of the arm in space, probably mapped in the primary somatosensory cortex, is available to parietal (Georgopoulos et al., 1984), motor (Georgopoulos et al., 1984; Kettner et al., 1988; Caminiti et al., 1990a), and premotor (Caminiti et al., 1991) cortices.

Neurons in the premotor cortex could perform "matching" operations since they receive both visuospatial (Kubota and Hamada, 1978; Godschalk et al., 1981; Rizzolatti et al., 1981b; Weinrich and Wise, 1982; Weinrich et al., 1984; Godschalk et al., 1985; Kurata and Tanji, 1986; Okano and Tanji, 1987; Riehle and Requin, 1989) and somatic (Rizzolatti et al., 1981a; Kurata and Tanji, 1986; Hummelsheim et al., 1988) information. The model also makes quantitative predictions: conversely to the synergy units, the activity of matching units is higher when the direction of the desired trajectory matches their directional tuning properties, independently from the initial position of the arm (which acts as a global gating signal). Consequently, their cell preferred direction should not rotate with the initial arm position. Such cells were not observed in premotor and motor cortices.

However, they could exist in the parietal cortex, and the use of the same experimental paradigm used in Caminiti et al. (1990a,b, 1991) will allow testing the validity of this hypothesis. In this respect, it is interesting to compare our results with those 
obtained in area 7, where visual parietal neurons have properties similar to those of matching units (Andersen and Mountcastle, 1983; Andersen et al., 1987; Zipser and Andersen, 1988): visual parietal neurons are tuned for a given retinal position of the visual stimulus, and their activity is gated by an eye position signal. This signal changes cell activity without changing the cell visual tuning.

Neurons sensitive to both visual and somatic information have been described in area 7 (Hyvarinen and Poranen, 1974; Leinonen et al., 1979); neurons active for both visual stimuli and hand movements have also been described in area 7 , with motor directional tuning properties similar to visual ones (Taira et al., 1990). However, it is important to note that somatomotor and visual information can be represented in area $7 \mathrm{a}$ in different neuronal functional classes (e.g., "visual fixation" and "projection" neurons) (Mountcastle et al., 1975). Neurons that project to matching units should be sensitive to the movement of the hand toward the fovea. In the parietal cortex, light-sensitive neurons exhibit properties such as foveal sparing and opponent vector organization; that is, they are insensitive to stimuli applied on the fovea and very sensitive to stimuli moving either inward toward the fovea or outward toward the periphery of the visual field (Motter and Mountcastle, 1981). These authors speculated that such populations of neurons could provide a neural basis for the central representation of "movements of objects in the periphery of the behavioral space when the eyes are fixed." Such neurons are therefore good candidates for monitoring the visual feedback produced by movements of the hand toward the fixation point and therefore toward the target when the eyes fixate the target.

An interesting alternative to a subdivision between matching and synergy units in two different cortices is the possibility that "matching" and "synergy" operations are performed in the same cortical area. In this case, they should be related by lateral connections that could provide the integration of the different matching opcrations leading to the same motor command. Since lateral connections between units that share common muscular targets are strong, these functional assemblies could trigger specific motor functions. Each cell of a functional assembly could integrate the results of the "matching" operations performed by other cells of the same assembly. These operations could be performed at the level of the premotor cortex since: (1) premotor neurons seem tuned for both visual and positional inputs as are matching units; (2) their preferred directions rotate with the initial position of the arm as for synergy units. In this case, the role of the parietal and motor cortices remains to be defined.

A more realistic view could be a "progressive match" model where visuomotor transformations are performed in a cooperative way in at least three areas (motor, premotor, and parietal areas) with three specific features in each one. (1) The matching interactions can be performed, at each step, between two sources of information. However, the precision of the visual information (and then the precision of the learned tuning) decreases from parietal to premotor and then to motor cortices. (2) The integration of several matching operations is provided both by lateral and feedforward interactions; conversely, the cumulative effect of these feedforward and lateral interactions increases from parietal to premotor and motor cortices. (3) The information signaling for outpul motor actions is provided both by lateral interactions in premotor and motor areas and by corollary discharges from motor to parietal areas (Kalaska et al., 1983). Such a gradient architecture has the advantage of minimizing the number of units having a prewired ability to perform a match between two sensory sources. An internal representation of the movement can be learned in parallel in such cooperative maps.

\section{Appendix A: Fundamental Correspondence between the 3-D External Space and the $n-D$ Internal Space}

Let $B O=\left(\mathbf{e}_{1}, \mathbf{e}_{2}, \mathbf{e}_{3}\right)$ be an orthonormal basis of the 3-D space and $B 1=\left(\mathbf{a}_{1}, \mathbf{a}_{2}, \ldots, \mathbf{a}_{n}\right)$ be a set of $n$ uniformly distributed 3$D$ vectors forming a generator set. Given a vector ${ }^{3} \mathbf{X}$ in $\mathbf{R}^{3}$ (coordinates ${ }^{3} X_{i}$ ), there exists a simple representation of any vector $\mathbf{X}$ in $B 1$ formed by its projection on the different vectors $\mathbf{a}_{i}$ composing $B 1$ :

$$
\begin{aligned}
& { }^{3} \mathbf{X}=\sum_{i}\left({ }^{3} \mathbf{X} \cdot \mathbf{e}_{i}\right) \mathbf{e}_{i}=\sum_{i}\left({ }^{3} \mathbf{X}_{i}\right) \mathbf{e}_{i}, \\
& { }^{3} \mathbf{X}=\frac{3}{n} \sum_{i}\left({ }^{3} \mathbf{X} \cdot \mathbf{a}_{i}\right) \mathbf{a}_{i} .
\end{aligned}
$$

If we define the vector ${ }^{n} \mathbf{X}={ }^{n} \mathbf{x}_{j}=(3 / n)^{3} \mathbf{X} \cdot \mathbf{a}_{j}, j=1, \ldots, n$ and $\mathbf{C}$ the $3 \times n$ matrix composed of the coefficients of the vectors of $B 1$ in the basis of $B 0$, it is easy to show that

$$
\begin{aligned}
{ }^{3} \mathbf{X} & =\mathbf{C}^{n} \mathbf{X} \text { (because } B 0 \text { is a basis), } \\
{ }^{n} \mathbf{X} & =\frac{3}{n} \mathbf{C}^{3} \mathbf{X}\left(\begin{array}{c}
\text { (because } B I \text { is uniformly } \\
\text { distributed) }
\end{array}\right. \\
\mathbf{C} \cdot{ }^{\prime} \mathbf{C} & =\frac{n}{3} \mathrm{I}, \\
{ }^{n} \mathbf{X} \cdot{ }^{n} \mathbf{X}^{\prime} & =\left(\frac{3}{n}\right)^{2} \mathbf{X} \cdot{ }^{3} \mathbf{X}^{\prime} \text { (from Eqs. A4 and A5). }
\end{aligned}
$$

\section{Appendix B}

\section{Activation rules}

We have modeled the operations performed by a cortical column in two steps, as shown in Figure 3 and described in Equations B1 and B2:

(1) Each layer division ( $z$ ) of a given processing unit first integrates inputs $\left(X_{y}^{z}\right)$ sharing a common origin. The activity of each layer division $\left(I^{z}\right)$ results from the combination of the distributed inputs weighted by specific coefficients $\left(w_{y}{ }^{z}\right)$ :

$I^{z}=\sum_{y} w_{y}^{z} X_{y}^{z}=\mathbf{W}^{z} \cdot \mathbf{X}^{z} \quad$ (vectors in bold character)

(2) The output of the unit $(A)$ depends upon the combination of two terms: the first term expresses the influence of each layer activity independently, with "layer" coefficients $\left(L^{z}\right)$, and the second one expresses the nonlinear interaction between two layers, with “interlayer" coefficients $\left(Q^{z / z^{\prime}}\right)$ :

$$
A=\sum_{z}\left(\sum_{z^{\prime}} Q^{z / z^{\prime}} I^{z^{\prime}}+L^{z}\right) I^{z}=\mathbf{I} \cdot \mathbf{Q I}+\mathbf{L} \cdot \mathbf{I} .
$$

\section{Learning rules}

Learning rules can change the three types of coefficients:

(1) "Intralayer" coefficients will be tuned by learning around values that correspond to the most probable input within this layer when the global output of the unit is strongly active, with a time delay $\tau$ corresponding to the external feedback loop between the strong output and the reafferent input: 
$W_{y}{ }^{z}=\operatorname{Prob}\left(X_{y}^{z}(t+\tau) \in E 2 /\{A(t) \in E 2\} \cap\left\{I^{z}(t+\tau) \in E 2\right\}\right)$.

(2) "Layer" coefficient will increase (to 1) if the corresponding input is always related to a previous strong output (this condition can occur when the output activity is always effective in reactivating, through an external feedback loop, the input arriving to this layer):

$$
\begin{gathered}
L^{z}=G\left[\operatorname{Prob}\left(I^{z}(\mathrm{t}+\tau) \in E 2 / A(t) \in E 2\right)\right] \\
\text { where } G[W]=2 W-1
\end{gathered}
$$

(3) "Interlayer" coefficients between two inputs will be close to 1 if each input has a low probability to be related to a strong output (low "layer" coefficient) and if their conjunction is always related to a strong output:

$$
Q^{z / z^{\prime}}=G\left[\operatorname{Prob}\left(I^{z}(t+\tau) \in E 2 /\{A(t) \in E 2\} \cap\left\{I^{z}(t) \in E 2\right\}\right)\right] .
$$

\section{References}

Abend W, Bizzi E, Morasso P (1982) Human arm trajectory formation. Brain 105:331-348.

Alexandre F, Guyot F, Haton JP, Burnod Y (1991) The cortical column, a new processing unit for multilayered networks. Neural Network 4:15-25.

Andersen RA, Mountcastle VB (1983) The influence of the angle of gaze upon the excitability of the light-sensitive neurons of the posterior parietal cortex. J Neurosci 3:532-548.

Andersen RA, Essick GK, Siegel RM (1987) Neurons of area 7 activated by both visual stimuli and oculomotor behavior. Exp Brain Res 67:316-322.

Arbib MA (1981) Perceptual structures and distributed motor control. In: Handbook of physiology - the nervous system. II. Motor control (Brouks VB, ed), pp 1449-1480. Ballinure: Willians and Wilkins.

Barruonuevo G, Brown TH (1983) Associative long-term potentiation in hippocampal slices. Proc Natl Acad Sci USA 80:7347-7351.

Bizzi E, Accornero N, Chapple W, Hogan N (1984) Posture control and trajectory formation during arm movement. J Neurosci 4:27362744.

Bullock D, Grossberg S (1988) Neural dynamics of planned arm movements: emergent invariance and speed accuracy properties during trajectory formation. Psychol Rev 95:49-90.

Burnod Y (1988) An adaptive neural network: the cerebral cortex. Paris: Masson.

Burnod Y, Dufossé M (1990) A model for the cooperation between the cerebellar and cerebral cortices in movement learning. In: Brain and space (Paillard J, ed), pp 446-460. Oxford: Oxford UP.

Caminiti P, Johnson PB, Urbano A (1990a) Making arm movements within different parts of space: dynamic aspects in the primate motor cortex. J Neurosci 10:2039-2058.

Caminiti R, Johnson PB, Burnod Y, Galli C, Ferraina S (1990b) Shifts of preferred directions of premotor cortical cells with arm movements performed across the work-space. Exp Brain Res 83:228-232.

Caminiti R, Johnson PB, Galli C, Ferraina S, Burnod Y (1991) Making arm movements within different parts of space: the premotor and motor cortical representation of a coordinate system for reaching to visual targets. J Neurosci 11:1182-1197.

Fortier PA, Kalaska JF, Smith AM (1989) Cerebellar neuronal activity related to wholc arm-reaching movements in the monkey. J Neurophysiol 62:198-211.

Fukushima K (1980) Neocognitron: a self-organizing neural network model for a mechanism of pattern recognition unaffected by shift in position. Biol Cybern 36:193-202.

Georgopoulos AP, Kalaska JF, Caminiti R, Massey JT (1982) On the relations between the direction of two-dimensional arm movements and cell discharge in primate motor cortex. J Neurosci 2:1527-1537.

Georgopoulos AP, Caminiti R, Kalaska JF, Massey JT (1983) Spatial coding of movement: a hypothesis concerning the coding of movement direction by motor cortical populations. Exp Brain Res [Suppl] $7: 327-336$.

Georgopoulos AP, Caminiti R, Kalaska, JF (1984) Static spatial effects in motor cortex and area 5: quantitative relations in 2-dimensional spacc. Exp Brain Res 54:446-454.

Georgopoulos AP, Kalaska JF, Caminiti R (1985) Relations between two-dimensional arm movements and single cells discharge in motor cortex and area 5: movement direction versus movement end-point. Exp Brain Res [Suppl] 10:175-183.

Georgopoulos AP, Schwartz AB, Kettner RE (1986) Neuronal population coding of movement direction. Science 233:1416-1419.

Georgopoulos AP, Kettner RE, Schwartz AB (1988) Primate motor cortex and free arm movements to visual targets in 3-dimensional space. II. Coding of the direction of movement by a neuronal population. J Neurosci 8:2928-2937.

Godschalk M, Lemon RN, Nijs HGT, Kuypers HGUM (1981) Behaviour of neurons in monkey peri-arcuate and precentral cortex before and during visually guided arm and hand movements. Exp Brain Res 44:113-116.

Godschalk M, Lemon RN, Kuypers HGUM, Van Der Sten J (1985) The involvement of monkey premotor cortex neurons in preparation of visually cued arm movements. Behav Brain Res 18:143-157.

Hay L (1984) The development of movement control. In: The psychology of human movement (Smyth M, Wing A, eds), pp 79-89. New York: Academic.

Hebb D (1949) The organization of behaviour. New York: Wiley.

Hein A (1971) Acquiring components of visually guided behavior. In: Minnesota symposia of child development (Pick A, ed), pp 53-68. Minneapolis, MN: University of Minnesota.

Held R, Hein A (1963) Movement produced stimulation in the development of visually guided behavior. J Comp Physiol Psychol 56: $872-876$

Hogan N (1985) The mechanics of multi-joints posture and movement. Biol Cybern 52:315-331.

Hogan N (1988) Planning and execution of multijoint movements. Can J Physiol Pharmacol 66:508-517.

Hollerbach JM, Atkeson CG (1987) Deducing planning variables from experimental arm trajectories: pitfalls and possibilities. Biol Cybern 56:279-292.

Hubel DH, Wiesel TN (1968) Receptive fields and functional architecture of monkey striate cortex. J Physiol (Lond) 195:215-243.

Hummelcheim $\mathrm{H}$, Bianchetti $\mathrm{M}$, Wiesendanger $\mathrm{M}$, Wiesendanger $\mathrm{R}$ (1988) Sensory inputs to agranular motor fields: a comparison betwcen precentral, supplementary motor and premotor areas in the monkey. Exp Brain Res 69:289-298.

Huntley JW, Jones EG (1991) Relationship of intrinsic connections to forelimb movement representations in monkey motor cortex: a correlative anatomic and physiological study. J Neurophysiol 66:390413 .

Hyvarinen J, Poranen A (1974) Function of the parietal associative area 7 as revealed from cellular discharges in alert monkeys. Brain 97:673-692.

Jupp PE, Mardia KV (1980) A general correlation coefficient for directional data and related regression problems. Biometrika 76:163173.

Kalaska JF, Caminiti R, Georgopoulos AP (1983) Cortical mechanisms related to the direction of two dimensional arm movements: relations in parietal area 5 and comparison with molor cortex. Exp Brain Res 51:247-260.

Kalaska JF, Cohen DAD, Hyde ML, Prud'homme M (1989) A comparison of movement direction-related versus load direction-related activity in primate motor cortex, using a two-dimensional reaching task. J Neurosci 9:2080-2102.

Kalaska JF, Cohen DAD, Prud'homme M, Hyde ML (1990) Parietal area 5 neuronal activity encodes movement kinematics, not movement dynamics. Exp Brain Res 80:351-364.

Kawato M, Furukawa K, Suzuki R (1987) A hierarchical neural network model for control and learning of voluntary movements. Biol Cybern 57:169-185.

Kawato M, Maeda Y, Uno Y, Suzuki R (1990) Trajectory formation of arm movements by cascade neural network models based on minimum torque change criterion. Biol Cybern 62:275-288.

Kettner RE, Schwartz AB, Georgopoulos AP (1988) Primate motor cortex and free-arm movements to visual targets in 3-dimensional space. II. Positional gradient and population coding of movement direction from various movement origins. J Neurosci 8:2938-2947.

Kubota K, Hamada I (1978) Visual tracking and neuron activity in the post-arcuate areas in the monkey. J Physiol (Paris) 74:297-312. 
Kuperstein M (1988a) Neural model of adaptive hand-eye coordination for single postures. Science 239:1308-1311.

Kuperstein M (1988b) An adaptive neural model for mapping invariant target position. Behav Neurosci 102:148-162.

Kurata K, Tanji J (1986) Premotor cortex neurons in macaque: activity before distal and proximal forelimb movements. J Neurosci $6: 403-$ 411.

Kwan HC, Murphy JT, Wong YC (1987) Interaction between neurons in precentral cortical zones controlling different joints. Brain Res 400: $259-269$

Lacquaniti F, Soechting JF (1982) Coordination of arm and wrist motion during a reaching task. J Neurosci 2:399-408.

Leinonen L, Hyvarinen J, Nyman G, Linnankoski I (1979) I. Functional properties of neurons in lateral part of associative area 7 in awake monkeys. Exp Brain Res 34:299-320.

Morasso P (1981) Spatial control of arm movements. Exp Brain Res 42:223-227.

Motter BC, Mountcastle VB (1981) The functional properties of lightsensitive neurons of the posterior parietal cortex studied in waking monkeys: foveal sparing and opponent vector organization. J Neurosci $1: 3-26$

Mountcastle VB (1978) An organizing principle for cerebral function: the unit module and the distributed system. In: The mindful brain (Edelman GM, Mountcastle VB, eds), pp 7-50. Cambridge, MA: MIT Press.

Mountcastle VB, Lynch JC, Georgopoulos A, Sakata H, Acuña C (1975) Posterior parietal association cortex of the monkey: command functions for operations within extrapersonal space. J Neurophysiol 38 : 871-908.

Nowak L, Bregestovski P, Ascher P, Herbet A, Prochiantz A (1984) Magnesium gates glutamate-activated channels in mouse central neurones. Nature 307:462-465.

Okano K, Tanji J (1987) Neuronal activities in the primate motor fields of the agranular frontal cortex preceding visually triggered and self-paced movements. Exp Brain Res 66:155-166.

Paillard J (1990) Knowing where and knowing how to get there. In: Brain and space (Paillard J, ed), pp 163-184. Oxford: Oxford UP.

Riehle A, Requin J (1989) Monkey primate motor and premotor cortex: single cell activity related to prior information about direction and extent of an intended movement. J Neurophysiol 61:534-549.

Rizzolatti G, Scandolara C, Gentilucci G, Matclli M, Gentilucci M (1981a) Afferent properties of peri-arcuate neurons in macaque mon- keys. I. Somatosensory responses. Behav Brain Res 2:125-146

Rizzolatti G, Scandolara C, Gentilucci G, Matelli M, Gentilucci M (1981b) Afferent properties of peri-arcuate neurons in macaque monkeys. II. Visual responses. Behav Brain Res 2:147-163.

Kumelhart DE, McClelland JL (1986) PDP models and general issues in cognitive science. In: Parallel distributed processing, explorations in the microstructure of cognition (Feldman JA, Hayes PJ, Rumelhart DE, eds), pp 110-146. Cambridge, MA: MIT Press.

Schwartz AB, Kettner RE, Georgopoulos AP (1988) Primate motor cortex and free arm movements to visual targets in three-dimensional space. I. Relations between single cell discharge and direction of movement. J Neurosci 8:2913-2927.

Sejnowski TJ (1986) Open questions about computation in cerebral cortex. In: Parallel distributed processing, explorations in the microstructure of cognition (Feldman JA, Hayes PJ, Rumelhart DE, eds), pp 372-389. Cambridge, MA: MIT Press.

Soechting JF, Flanders M (1989a) Sensorimotor representations for pointing to targets in three-dimensional space. J Neurophysiol 62: 582-594.

Soechting JF, Flanders M (1989b) Errors in pointing are due to approximations in sensorimotor transformations. J Neurophysiol 62: 595-608.

Soechting JF, Lacquaniti F (1981) Invariant characteristics of a pointing movement in man. J Neurosci 1:710-720.

Soechting JF, Terzuolo CA (1988) Sensorimotor transformations underlying the organization of arm movements in three-dimensional space. Can J Physiol Pharmacol 66:502-507.

Steinmetz MA, Motter BC, Duffy CJ, Mountcastle VB (1987) Functional properties of parietal visual neurons: radial organization of directionalities within the visual field. J Neurosci 7:177-191.

Swindale NV (1990) Is the cerebral cortex modular? Trends Neurosci 13:487-492.

Taira M, Mine S, Georgopoulos AP, Murata A, Sakata H (1990) Parietal cortex neurons of the monkey related to the visual guidance of hand movement. Exp Brain Res 83:29-36.

Weinrich M, Wise SP (1982) The premotor cortex of the monkey. J Neurosci 2:1329-1345.

Weinrich M, Wise SP, Mauritz KH (1984) A neurophysiological study of the premotor cortex in the rhesus monkey. Brain 107:385-414.

Zipser D, Andersen RA (1988) A back-propagation programmed network has simulated response properties of a subset of posterior parietal neurons. Nature 331:679-684. 